\title{
Construction of gene causal regulatory networks using microarray data with the coefficient of intrinsic dependence
}

\author{
Li-yu Daisy Liu ${ }^{1 *} \mathbb{0}$, Ya-Chun Hsiao ${ }^{1}$, Hung-Chi Chen², Yun-Wei Yang ${ }^{1}$ and Men-Chi Chang ${ }^{1}$
}

\begin{abstract}
Background: In the past two decades, biologists have been able to identify the gene signatures associated with various phenotypes through the monitoring of gene expressions with high-throughput biotechnologies. These gene signatures have in turn been successfully applied to drug development, disease prevention, crop improvement, etc. However, ignoring the interactions among genes has weakened the predictive power of gene signatures in practical applications. Gene regulatory networks, in which genes are represented by nodes and the associations between genes are represented by edges, are typically constructed to analyze and visualize such gene interactions. More specifically, the present study sought to measure gene-gene associations by using the coefficient of intrinsic dependence (CID) to capture more nonlinear as well as cause-effect gene relationships.

Results: A stepwise procedure using the CID along with the partial coefficient of intrinsic dependence ( $\mathrm{pCID}$ ) was demonstrated for the rebuilding of simulated networks and the well-known CBF-COR pathway under cold stress using Arabidopsis microarray data. The procedure was also applied to the construction of bHLH gene regulatory pathways under abiotic stresses using rice microarray data, in which OsbHLH104, a putative phytochrome-interacting factor (OsPIF14), and OsbHLH060, a positive regulator of iron homeostasis (OsPR/1) were inferred as the most affiliated genes. The inferred regulatory pathways were verified through literature reviews.

Conclusions: The proposed method can efficiently decipher gene regulatory pathways and may assist in achieving higher predictive power in practical applications. The lack of any mention in the literature of some of the regulatory event may have been due to the high complexity of the regulatory systems in the plant transcription, a possibility which could potentially be confirmed in the near future given ongoing rapid developments in bio-technology.
\end{abstract}

Keywords: Gene regulatory network, Cause-effect relationship, Microarray, Coefficient of intrinsic dependence

\section{Background}

Genes encode the information necessary for life, including the information determining an organism's molecular biology and ability to translate proteins directly involved in different biological activities. Therefore, the quantity of mRNA transcripts, or the expression levels of mRNA, mainly represent the gene activities in a biological system at the molecular level (Le Novère 2015). Using high-throughput gene profiling technologies that have undergone rapid development over the past several

\footnotetext{
${ }^{*}$ Correspondence: Iyliu@ntu.edu.tw

${ }^{1}$ Department of Agronomy, National Taiwan University, Taipei 106, Taiwan Full list of author information is available at the end of the article
}

decades, including microarray sequencing and nextgeneration sequencing, researchers are able to identify "gene signatures" defining genes whose expression levels are associated with particular traits or phenotypes under investigation (Ritchie et al. 2015). These gene signatures serve as biomarkers in a wide range of areas including drug development, disease diagnosis and prevention, and crop breeding, among others (Pérez-de-Castro et al. 2012; Gomez-Casati et al. 2013; Rykunov et al. 2016). Once such gene signatures have been recognized, questions such as "Do the gene signatures have synergistic interactions leading to the phenotypes?" and similar questions that presume gene-gene interactions that are 
well acknowledged in biosystems are commonly asked (Knight and Knight 2001; Segal et al. 2005). These questions can potentially be answered by simultaneously monitoring the expression levels of the regulators or regulatees using modern high-throughput gene expression technologies (Mantione et al. 2014; Liseron-Monfils and Ware 2015).

The Pearson correlation coefficient (PCC) is one of the mostly adopted methods for measuring the interactions among genes based on their expression levels (Song et al. 2012). Other measurements of association including the mutual information (MI) (Song et al. 2012), the partial Pearson correlation coefficient (pPCC) (de la Fuente et al. 2004), the coefficient of determination (CoD) (Higa et al. 2009), and the coefficient of intrinsic dependence (CID) (Liu et al. 2009) have also been used. The PCC and pPCC have the limitation of only identifying the linear relationship between any two gene expressions. In contrast, the CID requires neither distributional (e.g. normal) nor functional (e.g. linear) assumptions regarding gene expression data. $\mathrm{CID}(\mathrm{Y} \mid \mathrm{X})$ designates the CID value of a variable $\mathrm{Y}$ given the information of another variable $\mathrm{X}$. It takes any real value between 0 and +1 inclusive. It is +1 in the case of full dependence and is 0 in the case of independence. As the level of dependence increases, the CID value goes from 0 to 1 . In past studies, the CID has been used in conjunction with the correlation coefficient to construct an estrogen receptor regulatory network (Liu et al. 2009), to infer and classify co-regulatory events using two transcription factors (Liu et al. 2012), and to perform gene set association analysis (GSAA) (Tsai and Liu 2013). We have further demonstrated that the CID outperforms conventional methods for the identification of different association patterns (Liu et al. 2009; Tsai and Liu 2013).

After potential regulator-regulatee interactions under particular conditions have been identified, those interactions can then be connected to one another if they share the same genes. Such interactions are considered to constitute the small units of entire gene regulatory networks (GRNs), which may be combined together to form more comprehensive networks that better represent the biosystems in question (Liseron-Monfils and Ware 2015). An inferred GRN can therefore provide insights into the relationships between the genes of interest for specific experiments and clarify the understanding of biological functions involving complex biological phenomena (Karlebach and Shamir 2008). More specifically, an inferred GRN consisting of nodes (which represent the genes) and edges (which represent significant gene-gene interactions) reflects the gene regulation events that may concurrently or sequentially occur under the conditions being investigated. Previous studies have revealed that the edges between nodes in a GRN are typically not randomly allocated but are presumably assigned according to the scale-free topological model (e.g., Liseron-Monfils and Ware 2015). This would result in a network in which most nodes, with the exception of a few highly connected ones, are connected by a sparse number of edges. In this study, we focused on the inference and reconstruction of GRNs using the results of microarray experiments. More ambitiously, we sought to model the causal relationships in a single GRN.

Within a GRN, the relationship between a transcription factor (TF) and its target genes is usually expected to consist of a causal relationship (Pilpel et al. 2001). A directed edge pointing from the TF to the target gene would be specified in order to emphasize the origin (source) and the consequence (target) in this kind of relationship. Compared to a co-expression GRN (i.e., a network with undirected edges), a cause-and-effect GRN requires in vitro or in silico evidence to assign the direction to an edge (Simcha et al. 2013). However, in vitro evidence may not be available at all times, while the symmetric property of some commonly used statistics limits the exploration of causal effects in a GRN (Hsing et al. 2005). In this study, we utilized the asymmetric property of the $\mathrm{CID}$ (i.e., $\mathrm{CID}(\mathrm{Y} \mid \mathrm{X})$ is not necessarily equal to $\mathrm{CID}(\mathrm{X} \mid \mathrm{Y})$ ) to distinguish not only the associated gene pairs but the causes/effects in a gene regulation event. Asymmetry is a very unique feature of the CID, whereas some conventional methods, including the PCC, pPCC, and mutual information, provide symmetric results when considering the association between two variables. Other methods like the coefficient of determination may have limitations in terms of their capacity to be utilized on particular types of data (Liu 2005).

Another emphasis of this study was its utilization of a new measure derived from the CID to perform the stepwise selection of relevant genes for regulation path elongation. This new measure is called the partial coefficient of intrinsic dependence (pCID), a name which was inspired by the partial correlation coefficient (Hsiao and Liu 2016). The new measure was motivated by a difficulty encountered while using the multi-predictor CID described in a study by Liu et al. (2012) to identify relevant genes in the elongation step. Ideally, a proper stepwise procedure iteratively picks the relevant genes according to its magnitude of association to the target until no additional gene would significantly increase the amount of association. For example, CID(Source A|Target A1) would be significant while we also expect a significant $\mathrm{CID}$ (Source $\mathrm{A} \mid$ Target A1, Target A2) but an insignificant $\mathrm{CID}$ (Source $\mathrm{A} \mid$ Target $\mathrm{A} 1, \mathrm{X}$ ) given an irrelevant gene $\mathrm{X}$. However, due to the dominant effect of the most influential gene, i.e., Target A1, in the first step, 
CID(Source A|Target A1, X) would be mostly significant (Hsiao and Liu 2016). The pCID resolves this problem by decomposing only the information of the target variable which was not explained by the first predictor.

The present study further proposes a procedure to thoroughly reconstruct a GRN based on microarray gene expression data and using the CID along with the pCID. The procedure is first demonstrated on a simulated network. It is also applied to Arabidopsis microarray data to retrieve the CBF-COR pathway in Arabidopsis under cold stress in a "supervised" manner as well as to construct the rice bHLH gene regulatory network under abiotic stress in the seedling stage in an "unsupervised" manner. In the analysis of the CBF-COR pathway, it is known that cold-regulated genes (COR) are regulated by a family of transcription factors known as $\mathrm{C}$-repeat binding factors (CBFs), including the transcription factors $C B F 1, C B F 2$, and CBF3 (Fowler and Thomashow 2002; McKhann et al. 2008; Doherty et al. 2009). Experiments based on transgenic plants constitutively expressing $C B F 1, C B F 2$, and $C B F 3$ have suggested that the overexpression of the three genes induces the expression of similar gene sets, including COR47, COR6.6, and COR78 (Gilmour et al. 2004). Relatedly, RNA blot analyses have been conducted to confirm that the overexpression of $C B F 1$ and $C B F 3$ would induce COR15A, COR78, COR47, and COR6.6 gene expressions (Kasuga et al. 1999; Taji et al. 2002; Seki et al. 2002; Fowler and Thomashow 2002). McKhann et al. (2008) reported that the expression of COR15B may last for 5 weeks after cold treatment, while COR47 is only expressed within $24 \mathrm{~h}$ after cold treatment. By constructing the CBF-COR pathway in the present study, we examined the sensitivity of the proposed procedure and gained more biological insights about the possible synergistic behaviors among three CBFs.

In the construction of the rice bHLH gene regulatory network, a larger family of bHLH (basic helix-loophelix) transcription factors is of interest. The bHLH gene family in plants plays a principal role in developmental processes (Schaller 2012) that might govern the biotic and abiotic stress responses in plants (Fujita et al. 2006). However, the function of most rice bHLH genes remains unknown ( $\mathrm{Li}$ et al. 2006). OsbHLH001 (OsICE2) and OsbHLH002 (OsICE1) are induced at the protein level in response to cold and salt stresses, but they are not affected by cold stress at the mRNA level (Nakamura et al. 2011). Previous studies have shown that OsbHLH006 (RERJ1) is up-regulated in response to wounding and drought stresses (Kiribuchi et al. 2005); the expression of OsbHLH009 (OsMYC), a homolog of AtMYC2 in Arabidopsis, can be induced by drought stress (Baldoni et al. 2015); OsbHLH062 (OsbHLH1) could enhance cold tolerance (Li et al. 2006); OsbHLH148 is induced by salt stress and results in activation under cold stress (Seo et al. 2011); and OsbHLH152 (OsPILI1) could reduce internode elongation under drought stress (Todaka et al. 2012). In this study, we explored the responses of the OsbHLH genes and their potential target genes under abiotic stresses. We expect that the proposed procedure for reconstruct GRNs may be of assistance in reverse engineering biological pathways and better elucidating the understanding of bHLH gene regulatory processes.

\section{Methods \\ Coefficient of intrinsic dependence (CID) and partial coefficient of intrinsic dependence (pCID)}

The coefficient of intrinsic dependence, $\mathrm{CID}(\mathrm{Y} \mid \mathrm{X})$, quantifies the statistical dependence between two genes $(\mathrm{X}$, Y) observed from a sample of size $N$ by assessing the discrepancies between the conditional distribution of $\mathrm{Y}$, $\mathrm{F}(\mathrm{y} \mid \mathrm{x})$, given the values of $\mathrm{X}$ and the marginal distribution of $\mathrm{Y}, \mathrm{F}(\mathrm{y})$. The $\mathrm{CID}(\mathrm{Y} \mid \mathrm{X})$ value can be estimated from the sample using the following equation:

$$
\mathrm{CID}(Y \mid X)=\frac{1}{N} \times \frac{\sum_{i=1}^{N} \sum_{j=1}^{N}\left[\hat{F}\left(y_{i} \mid x_{j}\right)-\hat{F}\left(y_{i}\right)\right]^{2}}{\sum_{i=1}^{N} \hat{F}\left(y_{i}\right)\left[1-\hat{F}\left(y_{i}\right)\right]}
$$

where $x_{i}$ and $y_{i}$ are the observed value of $X$ and $Y$ in the $i$ th object, respectively, and the distribution functions were estimated by nonparametric kernel smoothing method using the "np" package in $\mathrm{R}$ (version 0.40-13) (Hayfield and Racine 2008).

Inspired by the partial correlation coefficient, the partial coefficient of intrinsic dependence (pCID) further decomposes the variability of the distribution of the variable $\mathrm{Y}$ which was not explained by the conditional distribution of the variable $\mathrm{Y}$ given the first variable $\mathrm{X}_{1}$ but can be explained after adding a second variable $\mathrm{X}_{2}$ (Hsiao and Liu 2016). When the two distribution functions are not identical, the discrepancy between them implies the amount of partial dependence between $X_{2}$ and $Y$ given $X_{1}$. Consequently, a recursive formula using CID values can be derived to compute the partial coefficient of intrinsic dependence of $\mathrm{Y}$ given $\mathrm{X}_{2}$ conditioned on $\mathrm{X}_{1}$ :

$$
\operatorname{pCID}\left(Y \mid X_{2} ; X_{1}\right)=\frac{\operatorname{CID}\left(Y \mid X_{2}, X_{1}\right)-\operatorname{CID}\left(Y \mid X_{1}\right)}{1-\operatorname{CID}\left(Y \mid X_{1}\right)} .
$$

The significance of the CID or pCID can then be assessed by the null distribution of the CID or pCID values by random permutations. That is, we randomly permuted the values of $Y$ and re-computed the CID or pCID values. This was repeated 1000 times and yielded 1000 internal control values of the CID values under independence. The $\mathrm{p}$ value for each association relationship 
between two variables of interest was determined by the number of values greater than or equal to the estimated CID or pCID divided by 1001 . Readers are referred to Hsiao and Liu (2016) for more mathematical details and toy examples for CID/pCID definitions as well as estimations.

\section{Strategy to construct the gene regulatory network}

The inference of a GRN has three steps (Fig. 1): (1) the identification of a significantly associated gene pair, (2) the regulation path elongation, and (3) the assembly of all the identified regulation paths. The basic principle of our GRN construction process designates gene $\mathrm{Y}$ as the source and gene $\mathrm{X}$ as the target, if $\mathrm{CID}(\mathrm{Y} \mid \mathrm{X})>\mathrm{CID}(\mathrm{X} \mid \mathrm{Y})$. When prior knowledge about the preferable source genes is lacking, any gene in the collected data can possibly be the source as well as the target. Due to the dramatic amount of genes simultaneously monitored in a microarray experiment, we developed the following heuristic approach for the first two steps. Starting from a source gene $T_{0}, \operatorname{CID}\left(T_{0} \mid T_{i}\right)$ is computed for one of the candidate target genes, $T_{i}$, where

$$
\begin{aligned}
& \operatorname{CID}\left(T_{0} \mid T_{i}\right) \\
& =\frac{1}{N} \times \frac{\sum_{j=1}^{N} \sum_{k=1}^{N}\left[\hat{F}\left(T_{0}=t_{j} \mid T_{i}=t_{k}\right)-\hat{F}\left(T_{0}=t_{j}\right)\right]^{2}}{\sum_{j=1}^{N} \hat{F}\left(T_{0}=t_{j}\right)\left[1-\hat{F}\left(T_{0}=t_{j}\right)\right]}
\end{aligned}
$$

$\hat{F}$ 's are the corresponding distribution functions estimated from the sample using the nonparametric kernel smoothing method (Hsiao and Liu 2016), and $t_{j}$ (and $t_{k}$ ) are the $j$ th (and $k$ th) realization of gene $T_{0}$ (and $T_{j}$ ) $(j$ or $k=1, \ldots, N)$. In order to reduce the computation required of the programming, we occasionally eliminated some irrelevant candidate target genes which caused the $\operatorname{CID}\left(T_{0} \mid T_{i}\right)$ values to be insignificant ( $\mathrm{p}$-value $>0.05$ ). Under these circumstances, the source gene $T_{0}$ will be discarded as the origin of a regulation path when all $\operatorname{CID}\left(T_{0} \mid T_{i}\right)$ values are insignificant in the first run.

In a set of $G$ genes, we first specify the source gene $T_{0}$. If $\operatorname{CID}\left(T_{0} \mid T_{(1)}\right)$ has the smallest p-value (or the largest CID value) among the results from all the candidate target genes, we connect the source gene $T_{0}$ and the target $T_{(1)}$. The direction is set from $T_{0}$ to $T_{(1)}$ if $\operatorname{CID}\left(T_{0} \mid T_{(1)}\right)$ is more significant than $\operatorname{CID}\left(T_{(1)} \mid T_{0}\right)$, or from $T_{(1)}$ to $T_{0}$, otherwise. The gene pair then proceeds to the elongation step. In the first step of elongation, $\operatorname{pCID}\left(T_{0} \mid T_{j}\right.$; $\left.T_{(1)}\right)$ and $\operatorname{pCID}\left(T_{(1)} \mid T_{j} ; T_{0}\right)$ are computed for one of the remaining candidate genes, $T_{j}$ (Fig. 2). If $\operatorname{pCID}\left(T_{0} \mid T_{(2)}\right.$; $T_{(1)}$ ) is the most significant outcome (that is, the one with the smallest significant $p$-value) among the results from all the candidate target genes, we connect the genes $T_{0}$ and $T_{(2)}$, with the direction being from $T_{0}$ to $T_{(2)}$ if the $p$-value of $\operatorname{pCID}\left(T_{0} \mid T_{(2)} ; T_{(1)}\right)<$ the $p$-value of $\operatorname{pCID}\left(T_{(2)} \mid T_{0} ; T_{(1)}\right)$, or from $T_{(2)}$ to $T_{0}$, otherwise. Instead, if $\operatorname{pCID}\left(T_{(1)} \mid T_{(2)} ; T_{0}\right)$ is the most significant outcome (that is, the one with the smallest significant $p$-value) among the results from all the candidate target genes, we connect the genes $T_{(1)}$ and $T_{(2)}$, with the direction being from $T_{(1)}$ to $T_{(2)}$ if $\operatorname{pCID}\left(T_{(1)} \mid T_{(2)} ; T_{0}\right)$ is more significant than $\mathrm{pCID}\left(T_{(2)} \mid T_{(1)} ; T_{0}\right)$, or from $T_{(2)}$ to $T_{(1)}$, otherwise. This completes the first run of the elongation. In the $k$ th run $(k \geq 2)$ of the elongation, all of the possible values of $\operatorname{pCID}\left(S \mid T_{j} ;\left\{T_{0}, T_{(1)}, \ldots, T_{(k)}\right\} \backslash\{S\}\right)$ for $S \in\left\{T_{0}, T_{(1)}, \ldots\right.$, a

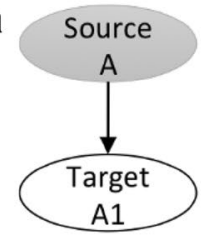

b

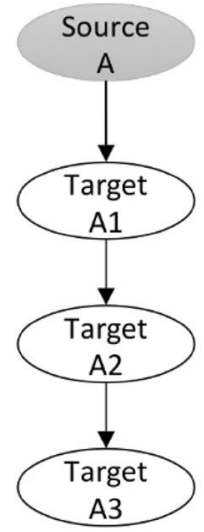

c

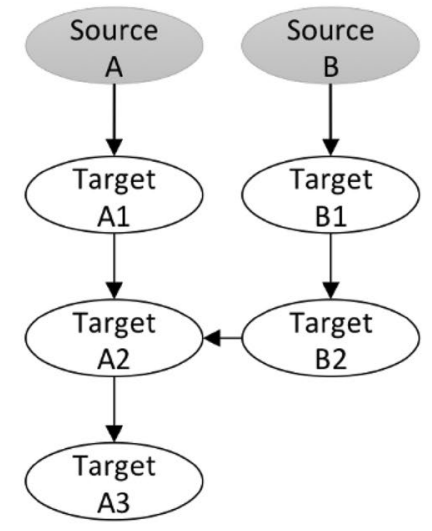

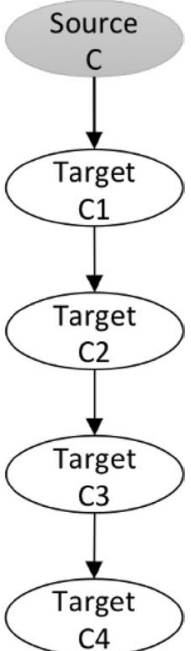

Fig. 1 Diagram of gene regulatory network inference workflow. a Identification of a significantly associated gene pair. $\mathbf{b}$ Regulation path elongation. c Assembly of all identified regulation paths 


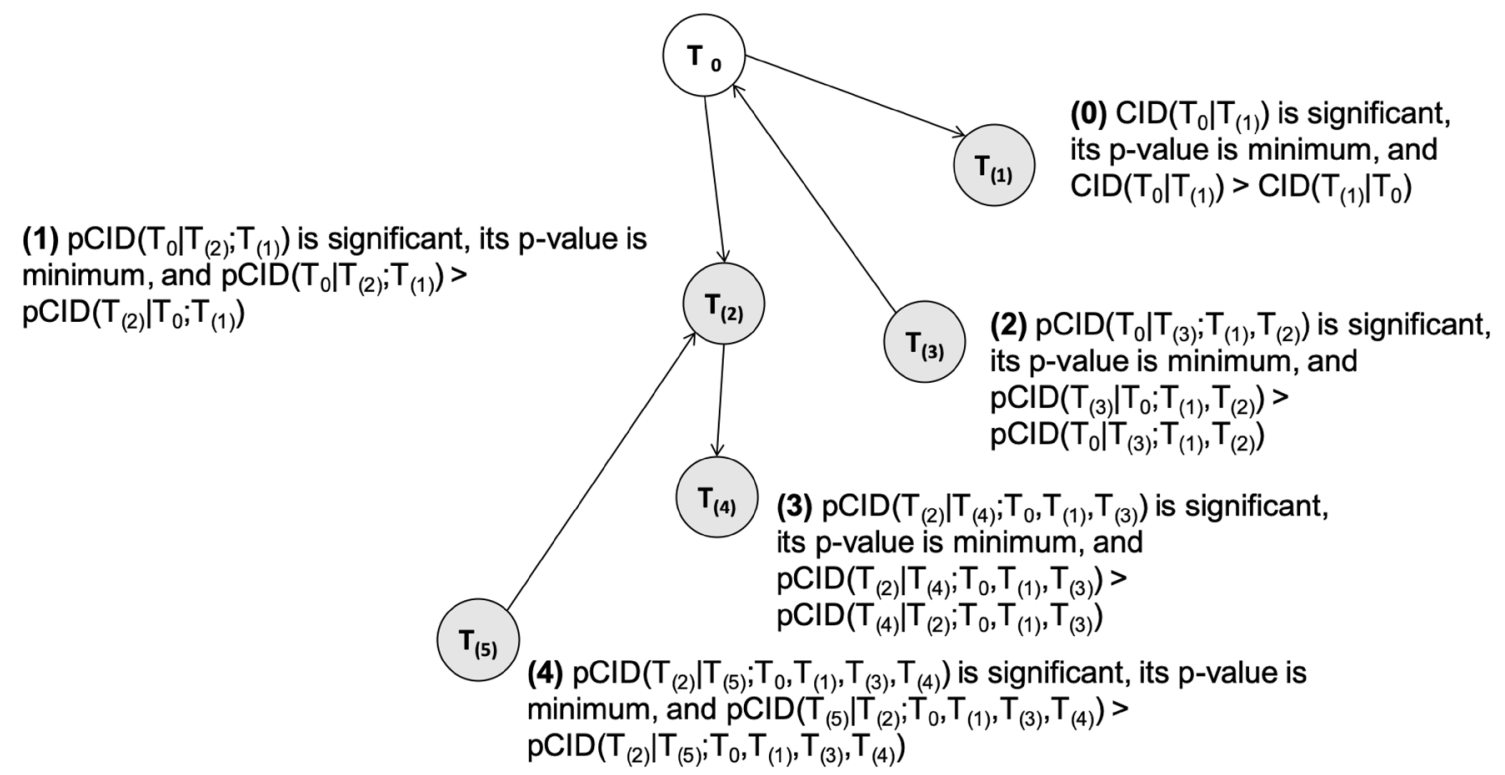

Fig. 2 Illustration of the heuristic approach for regulation path elongation

$\left.T_{(k)}\right)$ for the remaining $(G-k-1)$ genes are computed and result in $(k+1)(G-(k+1))$ pCID values. Suppose $\operatorname{pCID}\left(S \mid T_{(k+1)} ;\left\{T_{0}, T_{(1)}, \ldots, T_{(k)}\right\} \backslash\{S\}\right)$ is the most significant value, and we connect the node $S$ and $T_{(\mathrm{k}+1)}$; the direction is from $S$ to $T_{(\mathrm{k}+1)}$ if $\operatorname{pCID}\left(S \mid T_{(k+1)} ;\left\{T_{0}, T_{(1)}, \ldots\right.\right.$, $\left.\left.T_{(k)}\right\} \backslash\{S\}\right)$ is more significant than $\operatorname{pCID}\left(T_{(k+1)} \mid S ;\left\{T_{0}, T_{(1)}\right.\right.$, ..., $\left.\left.T_{(k)}\right\} \backslash\{S\}\right)$, or from $S$ to $T_{(\mathrm{k}+1)}$, otherwise. The elongation process continues until all of the $\operatorname{pCID}\left(S \mid T_{j} ;\left\{T_{0}, T_{(1)}\right.\right.$, ..., $\left.\left.T_{(e)}\right\} \backslash\{S\}\right)$ values are insignificant (p-value $>0.05$ ). The resulting network will contain $e+1$ nodes $\left(T_{0}, T_{(1)}, \ldots\right.$, $\left.T_{(e)}\right)$.

When the list of possible gene sources in the network is indicated by available biological evidence, the inference of the GRN can be simplified. In such cases, only $\operatorname{pCID}\left(S \mid T_{j} ;\left\{T_{0}, T_{(1)}, \ldots, T_{(k)}\right\} \backslash\{S\}\right)$ for $S \in$ the possible gene sources $\}$ and their p-values are computed in the kth run $(k \geq 2)$ of the elongation. The whole elongation process then continues until all of the $\operatorname{pCID}\left(S \mid T_{j} ;\left\{T_{0}, T_{(1)}\right.\right.$, ..., $\left.T_{(e)} \backslash \backslash\{S\}\right)$ values are insignificant (p-value $\left.>0.05\right)$ and result in a network with $e+1$ nodes $\left(T_{0}, T_{(1)}, \ldots, T_{(e)}\right)$.

\section{Simulation methods}

The proposed procedure of GRN inference was examined in a simulation study. A pseudo network with six nodes (genes) was generated according to a normal mixture model (Fig. 3a). It contained one source node (A11), four target nodes (A21, A22, A31 and A32), and one node (B) independent of the others. The expression levels of nodes A11 and B were randomly generated from the normal distribution with means and standard deviations both equal to $1, N(1,1)$. The expression levels of the target nodes were affected by two factors: the expression level and the binding efficiency of its direct source. This was intended to mimic the occasions in which (1) the transcription factor does not express so that the target gene is not regulated by the source gene, and (2) even if the source gene does express, the target gene may still not be regulated by the source gene due to the various binding efficiencies of the transcription factor. Let $S$ and $T$ denote the direct source and the target gene, respectively. In the simulated network, A11 was the direct source of $\{A 21, A 22\}$ and A21 was the direct source of $\{$ A31, A32\}. If the binding efficiency for this pair of $S$ and $T$ was set to be $100 b \%$, then $100(1-b) \%$ of the objects in the sample would not be affected by the expression level of $S$ and their expression levels would be generated from $\mathrm{N}(-1$, $1)$. The binding efficiency for $\{\mathrm{A} 11, \mathrm{~A} 21\},\{\mathrm{A} 11, \mathrm{~A} 22\}$, $\{A 21, A 31\}$, and $\{A 21, A 32\}$ were set to be 0.9, 0.7, 0.9, and 0.8 , respectively (Table 1 ). For the $100 b \%$ objects for which the regulation did take place, if the expression level of $S$ in the $i$ th sample was $s_{i}$, then the expression level of the $i$ th sample was randomly generated from $\mathrm{N}\left(s_{i}, 0.25\right)$ if $s_{i}>0$ and from $\mathrm{N}(-1,0.25)$ if $s_{i}<0$ (meaning that $S$ was not expressed). The pseudo network was replicated 100 times with sample sizes of $\mathrm{N}=25,50$, or 100 .

The approximate proportions of the gene expressions of the target gene actually determined by the expression levels of the source gene were expressed as $\mathrm{P}(S \rightarrow T)$. Because the target gene can only be regulated by the source gene if the expression level of the source gene 


\section{a Simulation setup}

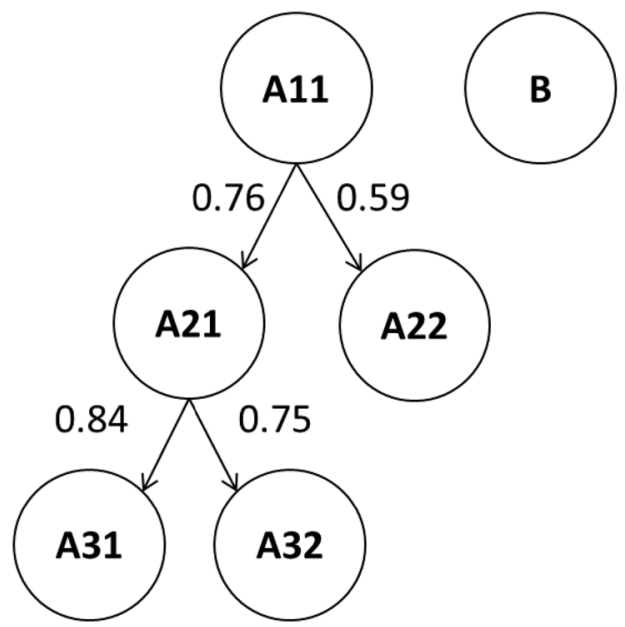

c Results of $n=50$

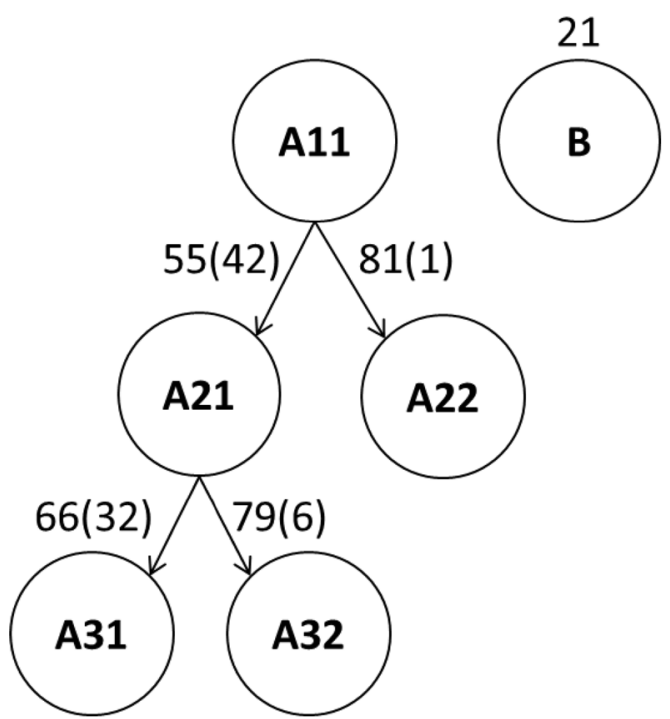

b Results of $n=25$

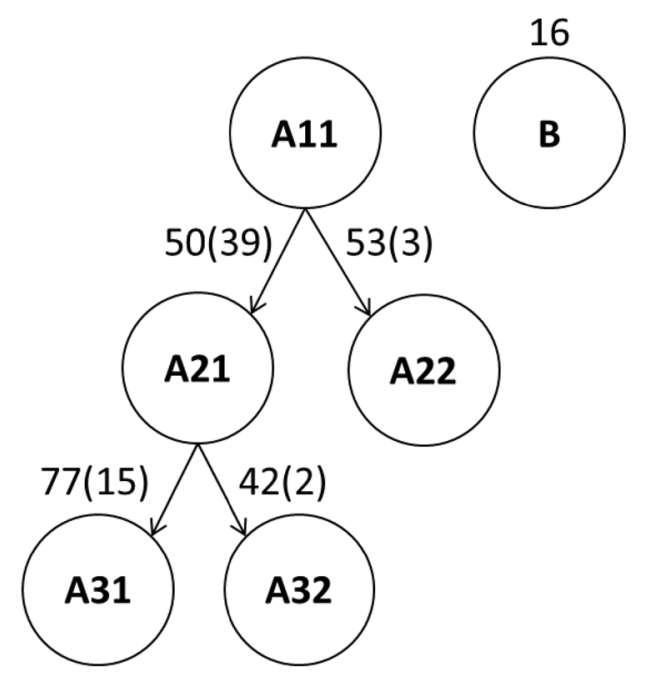

d Results of $n=100$

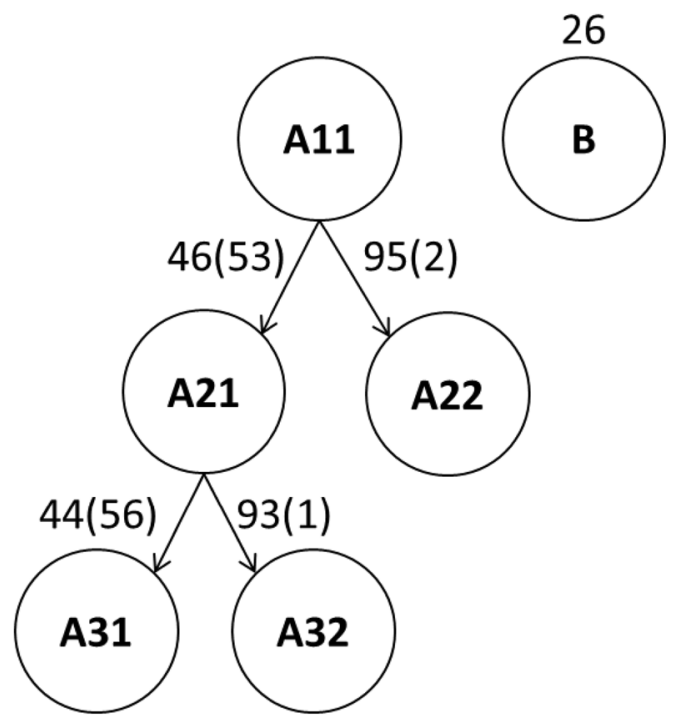

Fig. 3 Reconstruction of the a simulated network using sample size, $\mathbf{b} n=25, \mathbf{c} n=50$, and $\mathbf{d} n=100$. The numbers next to the arrows in $\mathbf{a}$ are the probabilities of the expression levels of the target genes actually determined by the expression levels of the source genes. The numbers next to the arrows in $\mathbf{b}-\mathbf{d}$ are the numbers of arrows pointing in correct directions (outside of the parentheses) and the numbers of arrows pointing in incorrect directions (in the parentheses)

is greater than 0 , we tabulated the two probabilities, $\mathrm{P}$ $(S \rightarrow T)$ and $\mathrm{P}(\mathrm{S}>0)$ denoting the probability that the expression level of the source gene is greater than 0 , for all combinations of $\{S, T\}$ in Table 1 . In the simulated network, we deliberately set different efficiencies of regulation for each pair of $\{S, T\}$ to examine the goodness of CID/pCID in detecting different levels of associations.

\section{Microarray expression data}

The first dataset used was the expression data of Arabidopsis thaliana under cold stress to study the well-known CBF-COR pathway. This dataset can be downloaded from the Arabidopsis Information Resource (TAIR) database (Garcia-Hernandez et al. 2002). This data originally consisted of 22,810 probes and 52 samples (submission number ME00325). The tissues were treated in a $4{ }^{\circ} \mathrm{C}$ 
Table 1 The binding efficiency (b) of the source gene (S) on the promoter region of the target gene (T), the probability that the expression level of the source gene is greater than $0, P(S>0)$, and the probability that the expression levels of the target gene are actually determined by the expression levels of the source gene, $P$ $(\mathrm{S} \rightarrow \mathrm{T})$, in the simulated network with 6 nodes (Fig. 3a)

\begin{tabular}{llll}
\hline$\{\boldsymbol{S}, \boldsymbol{T}\}$ & $\begin{array}{l}\text { Binding efficiency } \\
\text { (b) }\end{array}$ & $\mathbf{P}(\boldsymbol{S}>\mathbf{0})$ & $\mathbf{P}(\boldsymbol{S} \rightarrow \boldsymbol{T})$ \\
\hline$\{\mathrm{A} 11, \mathrm{~A} 21\}$ & 0.9 & 0.84 & 0.76 \\
$\{\mathrm{~A} 11, \mathrm{~A} 22\}$ & 0.7 & 0.84 & 0.59 \\
$\{\mathrm{~A} 21, \mathrm{~A} 31\}$ & 0.9 & 0.71 & 0.84 \\
$\{\mathrm{~A} 21, \mathrm{~A} 32\}$ & 0.8 & 0.71 & 0.75 \\
\hline
\end{tabular}

environment, and the expression levels were monitored after 0 (control), $0.5,1,3,6,12$, or $24 \mathrm{~h}$ of treatment. The microarray expression raw dataset was first subjected to pre-processing using the RMA (Robust Multichip Average) method (Irizarry et al. 2003) and was $\log 2$ transformed. As an instance of supervised study, only probes related to $\mathrm{CBF}-\mathrm{COR}$ regulation pathway in the microarray were collected for network construction according to their annotations.

A second dataset was used to study the bHLH pathway in rice (Oryza sativa). The expressions data can be downloaded from the NCBI-GEO database (Edgar et al. 2002) (accession numbers GSE6901 and GSE14275). The GSE6901 dataset includes the gene expressions of 7-dayold rice seedling samples under drought, salt, cold, and controlled conditions (three biological replicates of each condition). The GSE14275 dataset includes the gene expressions of 14-day-old rice seedling samples under heat and controlled conditions (three biological replicates of each condition). Expressed RNA samples were hybridized on Affymetrix microarrays (NCBI-GEO accession number GPL2025). The raw expression data of 51,279 probes from 18 samples were first subjected to pre-processing using the RMA method (Irizarry et al. 2003) and were $\log 2$ transformed. In this study, we were interested in the genes that were previously reported as related genes involved in the bHLH pathway (Li et al. 2006).

Some bHLH proteins recognize the G-box in the promoter region of their target genes (Gonzalez 2015). Among the bHLH-related genes, some of them can recognize and bind to the G-box according to Li et al. (2006). In this study, we also downloaded the gene sequences of the bHLH-related genes in the microarray from RAP-DB (version 7.0) (Sakai et al. 2013) to specify potential target genes containing G-box sequences in their promoter regions. The probes recognizing the bHLH-related genes and the probes containing G-box sequences were designated as the source and the candidate target genes, respectively, to construct the bHLH gene network. Note that there may have been some probes that served as both source and target genes since they not only could bind the G-box according to the literature but also had the G-box sequence in their promotor regions.

\section{Results}

Reconstruction of a pseudo network in the simulation

A pseudo network with six nodes (genes) was generated to assess the proposed procedure of GRN inference (Fig. 3a). Two source genes, A11 and B, were predetermined. The CID and pCID values as well as their p-values for a particular simulation of a sample with 25 realizations are shown in Table 2 as a demonstration of network reconstruction. Starting from A11, the results showed that $\mathrm{CID}(\mathrm{A} 11 \mid \mathrm{A} 21)$ had the largest value (0.2146) and the smallest p-value (0.0010), such that A21 was selected as the first node connected to A11. Because $\mathrm{CID}(\mathrm{A} 11 \mid \mathrm{A} 21)$ and $\mathrm{CID}(\mathrm{A} 21 \mid \mathrm{A} 11)$ had the same significant $p$-value (0.0010) and because the CID(A11|A21) value $(0.2146)$ was larger than the $C \operatorname{ID}(\mathrm{A} 21 \mid \mathrm{A} 11)$ value (0.1905), the direction was set from A11 to A21. The computation of $\mathrm{pCID}(\mathrm{A} 11 \mid \mathrm{x} ; \mathrm{A} 21)$ and $\mathrm{pCID}(\mathrm{A} 21 \mid \mathrm{x}$; A11) for another gene $x$ followed and resulted in the selection of A22 as the second node connected to A11 due to the fact that pCID(A11|A22; A21) had the smallest $\mathrm{p}$-value $(0.0010)$ and the largest $\mathrm{pCID}$ value (0.1679). The direction was set from A11 to A22 because $\mathrm{pCID}(\mathrm{A} 11 \mid \mathrm{A} 22 ; \mathrm{A} 21)$ had the same significant $p$-value (0.0010) as $\mathrm{pCID}(\mathrm{A} 22 \mid \mathrm{A} 11 ; \mathrm{A} 21)$ but $\mathrm{pCID}(\mathrm{A} 11 \mid \mathrm{A} 22$; $\mathrm{A} 21)=0.1679>\mathrm{pCID}(\mathrm{A} 22 \mid \mathrm{A} 11 ; \quad \mathrm{A} 21)=0.1460 . \quad$ Similarly, the third target and fourth target, $\mathrm{A} 31$ and $\mathrm{A} 32$, were selected based on $\mathrm{pCID}(\mathrm{A} 21 \mid \mathrm{A} 31 ; \mathrm{A} 11, \mathrm{~A} 22)$ and pCID(A21|A32; A11, A22, A31); both A31 and A32 were connected from A21 due to pCID(A21|A31; A11, $\mathrm{A} 22)=0.1751>\mathrm{pCID}(\mathrm{A} 31 \mid \mathrm{A} 21 ; \quad \mathrm{A} 11, \quad \mathrm{~A} 22)=0.1707$ (both had the same $p$-value) and [ $p$-value of $\mathrm{pCID}(\mathrm{A} 21 \mid \mathrm{A} 32 ; \mathrm{A} 11, \mathrm{~A} 22, \mathrm{~A} 31)]=0.0070<[p$-value of $\mathrm{pCID}(\mathrm{A} 32 \mid \mathrm{A} 11 ; \mathrm{A} 11, \mathrm{~A} 22, \mathrm{~A} 32)]=0.8392$. When considering the negative-control node $B$ as the source node, it had all insignificant values of CID at the first step of GRN inference and was isolated from the other nodes. Therefore, the resulting network was identical to our setting shown in Fig. 3a.

We collected all the networks reconstructed in the simulations for $\mathrm{N}=25,50$, and 100; networks consisting of the same set of nodes were grouped together and the groups that occurred at least 5 times are shown in Additional file 1: Figure S1. There were 14, 65, and 81 of 100 reconstructed networks that successfully recovered the correct network structure in simulations for $\mathrm{N}=25$, 50, and 100, respectively. Moreover, 54 and 10 of 100 reconstructed networks only correctly revealed the 
Table 2 The estimated CID and pCID values in one simulation of sample size $\mathbf{n}=\mathbf{2 5}$

\begin{tabular}{|c|c|c|c|}
\hline CID/pCID & Estimated (p-value) & $\mathrm{CID} / \mathrm{pCID}$ & Estimated ( $p$-value) \\
\hline $\mathrm{CID}(\mathrm{A} 11 \mid \mathrm{A} 21)$ & $0.2146(0.0010)$ & $\mathrm{CID}(\mathrm{A} 21 \mid \mathrm{A} 11)$ & $0.1905(0.0010)$ \\
\hline $\mathrm{CID}(\mathrm{A} 11 \mid \mathrm{A} 22)$ & $0.2039(0.0010)$ & & \\
\hline $\mathrm{CID}(\mathrm{A} 11 \mid \mathrm{A} 31)$ & $0.1019(0.0060)$ & & \\
\hline $\mathrm{CID}(\mathrm{A} 11 \mid \mathrm{A} 32)$ & $0.0425(0.0949)$ & & \\
\hline $\mathrm{CID}(\mathrm{A} 11 \mid \mathrm{B})$ & $0.0534(0.0639)$ & & \\
\hline $\mathrm{pCID}(\mathrm{A} 11 \mid \mathrm{A} 22 ; \mathrm{A} 21)$ & $0.1679(0.0010)$ & $\mathrm{pCID}(\mathrm{A} 22 \mid \mathrm{A} 11 ; \mathrm{A} 21)$ & $0.1460(0.0030)$ \\
\hline $\mathrm{pCID}(\mathrm{A} 11 \mid \mathrm{A} 31 ; \mathrm{A} 21)$ & $0.0288(0.5075)$ & & \\
\hline $\mathrm{pCID}(\mathrm{A} 11 \mid \mathrm{A} 32 ; \mathrm{A} 21)$ & $0.0315(0.4575)$ & & \\
\hline $\mathrm{pCID}(\mathrm{A} 11 \mid \mathrm{B} ; \mathrm{A} 21)$ & $0.0133(0.6444)$ & & \\
\hline $\mathrm{pCID}(\mathrm{A} 21 \mid \mathrm{A} 22 ; \mathrm{A} 11)$ & $0.0027(0.8462)$ & & \\
\hline pCID(A21|A31; A11) & $0.1581(0.0010)$ & & \\
\hline $\mathrm{pCID}(\mathrm{A} 21 \mid \mathrm{A} 32 ; \mathrm{A} 11)$ & $0.0934(0.0120)$ & & \\
\hline $\mathrm{pCID}(\mathrm{A} 21 \mid \mathrm{B} ; \mathrm{A} 11)$ & $0.0363(0.2957)$ & & \\
\hline $\mathrm{pCID}(\mathrm{A} 11 \mid \mathrm{A} 31 ; \mathrm{A} 21, \mathrm{~A} 22)$ & $0.0285(0.0889)$ & & \\
\hline pCID(A11|A32; A21, A22) & $0.0129(0.3247)$ & & \\
\hline $\mathrm{pCID}(\mathrm{A} 11 \mid \mathrm{B} ; \mathrm{A} 21, \mathrm{~A} 22)$ & $-0.0010(0.6114)$ & & \\
\hline $\mathrm{pCID}(\mathrm{A} 21 \mid \mathrm{A} 31 ; \mathrm{A} 11, \mathrm{~A} 22)$ & $0.1751(0.0020)$ & $\mathrm{pCID}(\mathrm{A} 31 \mid \mathrm{A} 21 ; \mathrm{A} 11, \mathrm{~A} 22)$ & $0.1707(0.0020)$ \\
\hline $\mathrm{pCID}(\mathrm{A} 21 \mid \mathrm{A} 32 ; \mathrm{A} 11, \mathrm{~A} 22)$ & $0.1001(0.0559)$ & & \\
\hline $\mathrm{pCID}(\mathrm{A} 21 \mid \mathrm{B} ; \mathrm{A} 11, \mathrm{~A} 22)$ & $0.0523(0.3187)$ & & \\
\hline $\mathrm{pCID}(\mathrm{A} 22 \mid \mathrm{A} 31 ; \mathrm{A} 11, \mathrm{~A} 21)$ & $0.0007(0.8961)$ & & \\
\hline $\mathrm{pCID}(\mathrm{A} 22 \mid \mathrm{A} 32 ; \mathrm{A} 11, \mathrm{~A} 21)$ & $0.0059(0.7872)$ & & \\
\hline $\mathrm{pCID}(\mathrm{A} 22 \mid \mathrm{B} ; \mathrm{A} 11, \mathrm{~A} 21)$ & $-0.0036(0.9171)$ & & \\
\hline $\mathrm{pCID}(\mathrm{A} 11 \mid \mathrm{A} 32 ; \mathrm{A} 21, \mathrm{~A} 22, \mathrm{~A} 31)$ & $-0.0019(0.7772)$ & & \\
\hline $\mathrm{pCID}(\mathrm{A} 11 \mid \mathrm{B} ; \mathrm{A} 21, \mathrm{~A} 22, \mathrm{~A} 31)$ & $-0.0055(0.7972)$ & & \\
\hline $\mathrm{pCID}(\mathrm{A} 21 \mid \mathrm{A} 32 ; \mathrm{A} 11, \mathrm{~A} 22, \mathrm{~A} 31)$ & $0.1165(0.0070)$ & $\mathrm{pCID}(\mathrm{A} 32 \mid \mathrm{A} 21 ; \mathrm{A} 11, \mathrm{~A} 22, \mathrm{~A} 31)$ & $0.0768(0.8392)$ \\
\hline $\mathrm{pCID}(\mathrm{A} 21 \mid \mathrm{B} ; \mathrm{A} 11, \mathrm{~A} 22, \mathrm{~A} 31)$ & $0.0272(0.7772)$ & & \\
\hline $\mathrm{pCID}(\mathrm{A} 22 \mid \mathrm{A} 32 ; \mathrm{A} 11, \mathrm{~A} 21, \mathrm{~A} 31)$ & $0.0147(0.7692)$ & & \\
\hline $\mathrm{pCID}(\mathrm{A} 22 \mid \mathrm{B} ; \mathrm{A} 11, \mathrm{~A} 21, \mathrm{~A} 31)$ & $0.0008(0.9411)$ & & \\
\hline $\mathrm{pCID}(\mathrm{A} 31 \mid \mathrm{A} 32 ; \mathrm{A} 11, \mathrm{~A} 21, \mathrm{~A} 22)$ & $0.0523(0.2567)$ & & \\
\hline $\mathrm{pCID}(\mathrm{A} 31 \mid \mathrm{B} ; \mathrm{A} 11, \mathrm{~A} 21, \mathrm{~A} 22)$ & $0.0196(0.8192)$ & & \\
\hline $\mathrm{pCID}(\mathrm{A} 11 \mid \mathrm{B} ; \mathrm{A} 21, \mathrm{~A} 22, \mathrm{~A} 31, \mathrm{~A} 32)$ & $0.0182(0.3596)$ & & \\
\hline $\mathrm{pCID}(\mathrm{A} 21 \mid \mathrm{B} ; \mathrm{A} 11, \mathrm{~A} 22, \mathrm{~A} 31, \mathrm{~A} 32)$ & $0.0257(0.7453)$ & & \\
\hline $\mathrm{pCID}(\mathrm{A} 22 \mid \mathrm{B} ; \mathrm{A} 11, \mathrm{~A} 21, \mathrm{~A} 31, \mathrm{~A} 32)$ & $0.0023(0.9610)$ & & \\
\hline $\mathrm{pCID}(\mathrm{A} 31 \mid \mathrm{B} ; \mathrm{A} 11, \mathrm{~A} 21, \mathrm{~A} 22, \mathrm{~A} 32)$ & $0.0156(0.8611)$ & & \\
\hline $\mathrm{pCID}(\mathrm{A} 32 \mid \mathrm{B} ; \mathrm{A} 11, \mathrm{~A} 21, \mathrm{~A} 22, \mathrm{~A} 31)$ & $0.0368(0.7203)$ & & \\
\hline $\mathrm{CID}(\mathrm{B} \mid \mathrm{A} 11)$ & $0.0273(0.6613)$ & & \\
\hline $\mathrm{CID}(\mathrm{B} \mid \mathrm{A} 21)$ & $0.0112(0.6394)$ & & \\
\hline $\mathrm{CID}(\mathrm{B} \mid \mathrm{A} 22)$ & $0.0321(0.2078)$ & & \\
\hline $\mathrm{CID}(\mathrm{B} \mid \mathrm{A} 31)$ & $0.0127(0.5784)$ & & \\
\hline $\mathrm{CID}(\mathrm{B} \mid \mathrm{A} 32)$ & $0.0204(0.3686)$ & & \\
\hline
\end{tabular}

For simplicity, only the results of two pre-determined source genes, $\mathrm{A} 11$ and $\mathrm{B}$, are shown Italic signifies the combination having the largest $C I D / p C I D$ value and the smallest $p$-value

partial network for $\mathrm{N}=25$ and 50, respectively. A22 and A32 were discarded most often in the partial networks under the sample size $\mathrm{N}=25$ due to their lower proportions $(59 \%$ and $0.76 \times 0.75=57 \%$, respectively) of gene expressions actually determined by the expression levels of A11 (Fig. 3a). Similarly, the edges A11-A22 and A21-A32 would be occasionally discarded under the sample size $N=50$. The GRN could be mostly accurately reconstructed under the sample size $\mathrm{N}=100$.

In Fig. $3 \mathrm{~b}-\mathrm{d}$, the numbers of all the connections between two nodes from 100 simulations under $\mathrm{N}=25$, 
50 and, 100 are shown. When the sample size $\mathrm{N}=25$ and the source node was A11, there were $89 \%$ of networks that connected A11-A21 together, 92\% of networks that connected A21-A31, 56\% of networks that connected A11-A22, and 44\% of networks that connected A21-A32, while $16 \%$ of the networks included the negative control node, $\mathrm{B}$ (Fig. $3 \mathrm{~b}$ ). When $\mathrm{N}=50$, $97 \%, 98 \%, 82 \%$, and $85 \%$ of the networks contained the edges A11-A21, A21-A31, A11-A22, and A21-A32, respectively, while $21 \%$ of them had the negative control node, B (Fig. 3c). When $\mathrm{N}=100,99 \%, 100 \%, 97 \%$, and $94 \%$ of the networks contained the edges A11A21, A21-A31, A11-A22, and A21-A32, respectively, while $26 \%$ of them had the negative control node, B
(Fig. 3d). When the negative control node, B, was set to be the source gene, 16\% (Fig. 3b), 21\% (Fig. 3c), and $26 \%$ (Fig. 3d) of the networks were significantly built at $\alpha=0.05$.

\section{Analysis of CBF-COR pathway under cold stress in Arabidopsis thaliana}

Using the microarray data of 44 samples, we intended to reconstruct the CBF-COR gene regulatory network (GRN) of eight genes related to cold stress in Arabidopsis. Three CBF TFs took turns being the source of the regulation path elongation, while the other probes were all considered as potential targets. Figure $4 \mathrm{~b}-\mathrm{d}$ present the reconstructed paths from the source CBF genes

\section{a}

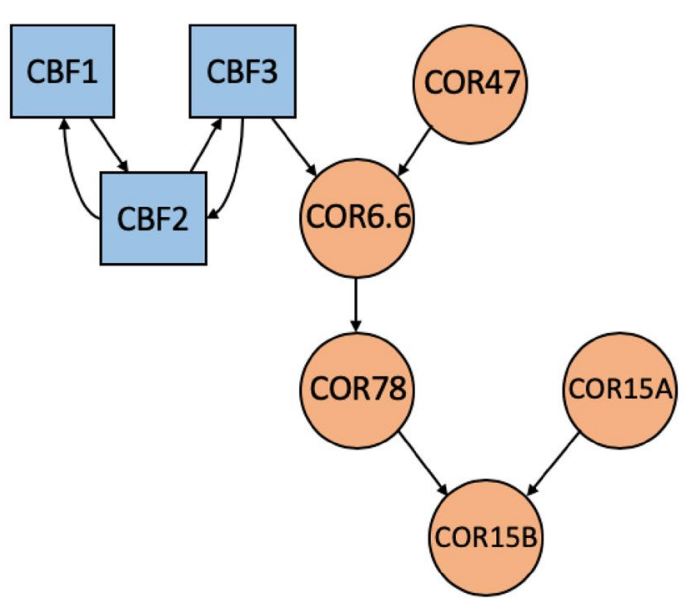

b

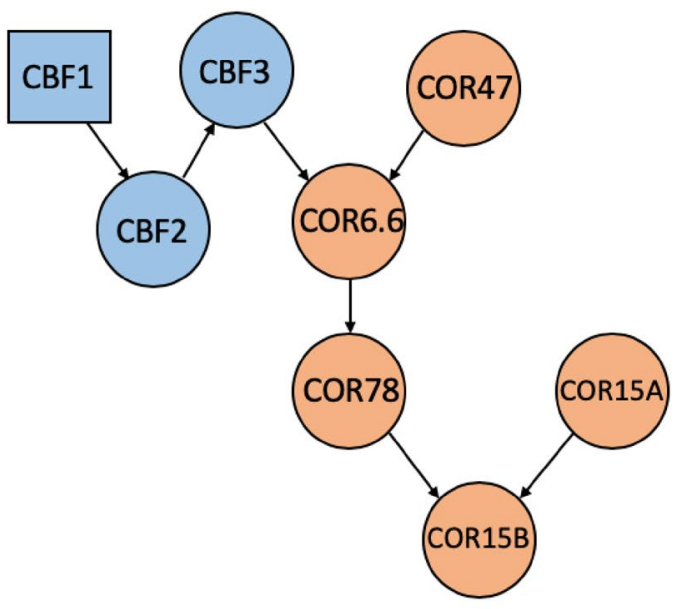

d

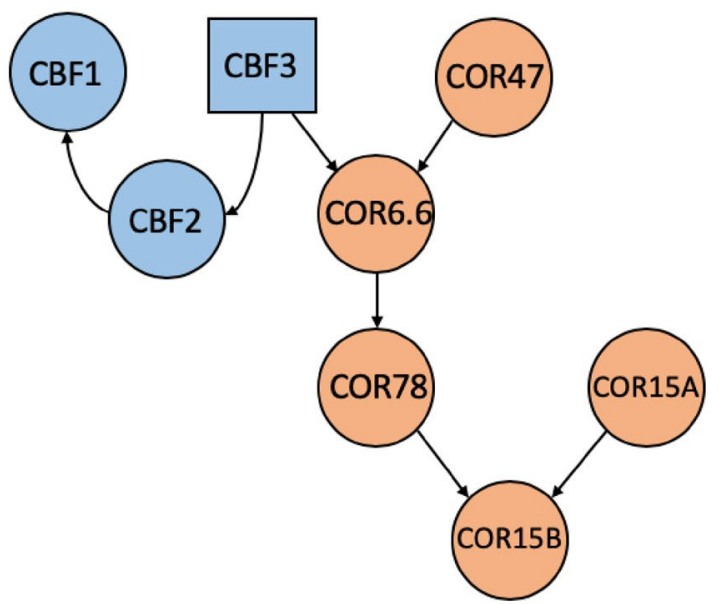

Fig. 4 The reconstructed paths from the source CBF genes (blue rectangles) to the target CBF genes (blue circles) or target COR genes (orange circles), respectively, starting from $\mathbf{b} C B F 1, \mathbf{c} C B F 2$, and $\mathbf{d} C B F 3$. All the paths in $\mathbf{b}-\mathbf{d}$ were combined to reconstruct the CBF-COR GRN in $\mathbf{a}$ 
(blue rectangles) to the target $\mathrm{CBF}$ genes (blue circles) or the target COR genes (orange circles), respectively. The resulting paths starting from $C B F 2$ (Fig. 4c) and $C B F 3$ (Fig. 4d) were identical; the paths starting from $C B F 1$ (Fig. 4b) were similar to them except that the directions of the arrows between the CBF genes were opposite. We combined these paths to reconstruct the CBF-COR GRN shown in Fig. 4a. Both CBF1 and CBF3 were connected with $C B F 2$ in the GRN, while $C B F 3$ had direct contact with the studied downstream COR genes. The COR6.6 was the first receiver of the information passed down from the CBF genes, which further influenced COR78 and COR15B. In contrast, COR47 and COR15A served as signal providers in the GRN.

\section{Construction of rice bHLH gene regulatory network under abiotic stress}

The 61 known bHLH genes (72 probes) capable of binding to G-box sequences according to the literature (Gonzalez 2015) were assigned as source genes of the network and the 104 bHLH probes containing G-box sequences in their promoter regions were recruited as the potential targets. There were 54 probes that could be either sources or targets (Additional file 2: Table S1). All the sub-networks from all 72 source probes were assembled together to form the final version of the bHLH GRN in this study (Fig. 5).

We considered the source gene, OsbHLH104-1, to illustrate the elongation process in the bHLH GRN construction (Fig. 1b). Among those CID values of OsbHLH104-1 given to all 104 target probes, the largest one was CID(OsbHLH104-1| OsbHLH104-2) $=0.3926$. Next, the largest significant pCID value was pCID(OsbHLH1041 OsbHLH139-1; OsbHLH104-2) $=0.0738$, provided that the OsbHLH104-1 was the source and OsbHLH104-2 was the first target, implying that OsbHLH139-1 was the second target in this particular sub-network. The elongation process stopped due to the fact that OsbHLH139-1 can be a target variable but not a source variable. When considering another source gene, OsbHLH056-1, the elongation process was stopped after adding one target gene, OsbHLH025-1, since all of the

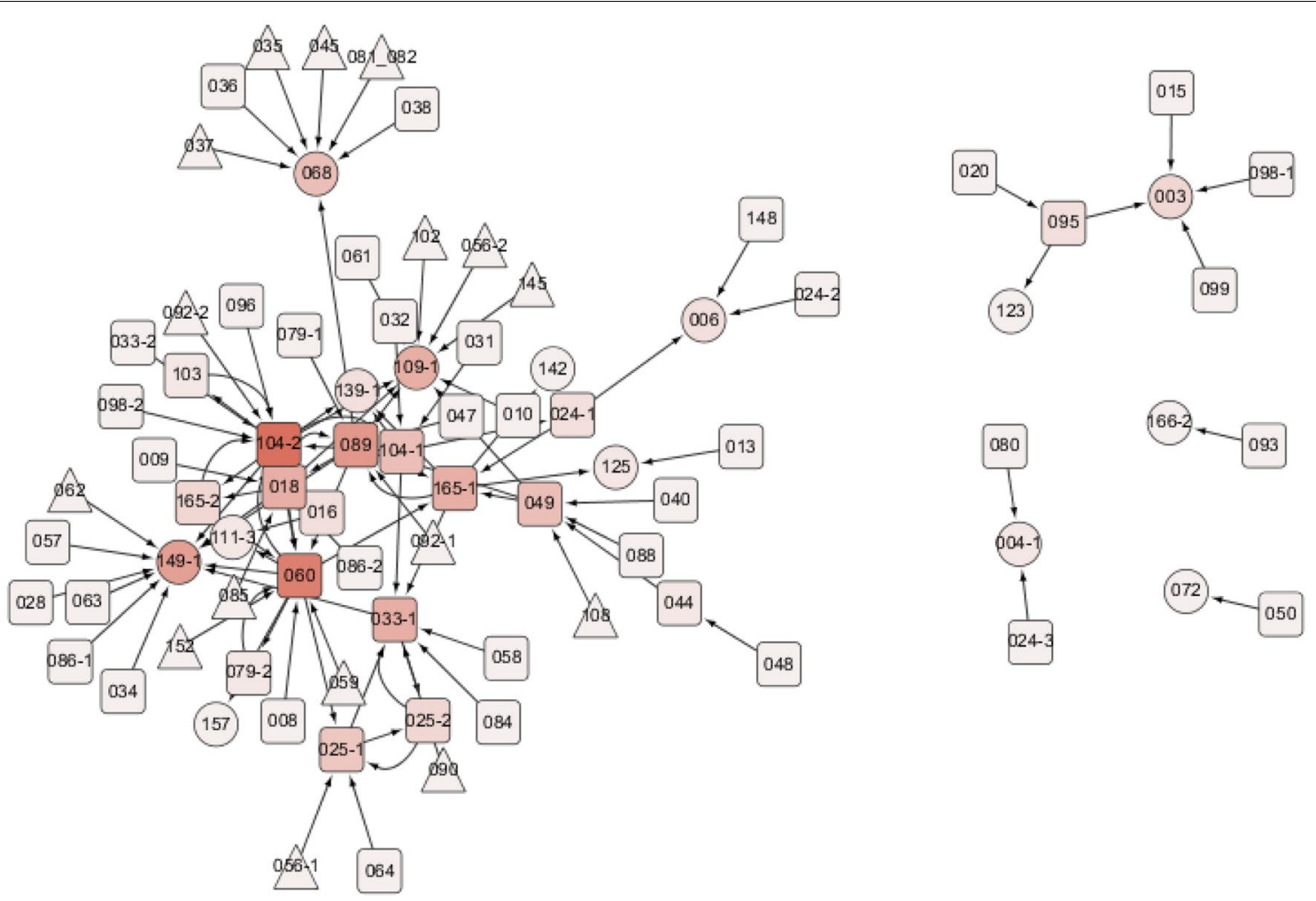

Fig. 5 Triangle nodes indicate the bHLH probes capable of binding to G-box sequences (G-box binders) but not having G-box sequences in their promoter regions (being sources only); ellipse nodes indicate the bHLH probes having G-box sequences in their promoter regions but not known as the G-box binders (being targets only); round rectangle nodes are the G-box binders having G-box sequences in their promoter regions (being both sources and targets). The shade of the fill color in the node represents the total degree of the node. A list of all the probes (nodes) used in the study is provided in Additional file 2: Table S1, and the sub-network for each source probe is in Additional file 2: Table S2 
pCID(OsbHLH056-1|T; OsbHLH025-1) values were insignificant ( $\mathrm{p}$-value $>0.05$ ). All 72 source probes were processed using the same criteria (stopping when either encountering a target-only gene or having all insignificant $\mathrm{CID} / \mathrm{pCID}$ values), and their resulting sub-networks are provided in Supp Table S2. Three of the 72 sources (OsbHLH083, OsbHLH144, and OsbHLH135) did not have significant CID values and their sub-networks were not further extended. Half of the 72 sub-networks expanded to only one target from the source; 28 sub-networks expanded to two targets; and 6 sub-networks expanded to three or four targets.

\section{Discussion}

The simulations verified the sensitivity and specificity of detecting directed gene-gene association by using CID/ pCID

The medians and interquartile ranges of some CID and pCID values summarized from the 100 simulations are shown in Table 3. The CID values of A11 to a directed or undirected

Table 3 Summary of estimated CID and pCID values in 100 simulations

\begin{tabular}{|c|c|c|c|c|c|c|}
\hline & \multicolumn{2}{|l|}{$n=25$} & \multicolumn{2}{|l|}{$n=50$} & \multicolumn{2}{|l|}{$n=100$} \\
\hline & Median (IQR) & Sig. prop. & Median (IQR) & Sig. prop. & Median (IQR) & Sig. prop. \\
\hline $\mathrm{CID}(\mathrm{A} 11 \mid \mathrm{A} 21)$ & $0.197(0.053)$ & 1.00 & $0.205(0.053)$ & 1.00 & $0.232(0.038)$ & 1.00 \\
\hline $\mathrm{CID}(\mathrm{A} 11 \mid \mathrm{A} 22)$ & $0.110(0.057)$ & 0.86 & $0.123(0.052)$ & 1.00 & $0.140(0.033)$ & 1.00 \\
\hline $\mathrm{CID}(\mathrm{A} 11 \mid \mathrm{A} 31)$ & $0.135(0.063)$ & 0.93 & $0.146(0.061)$ & 1.00 & $0.160(0.035)$ & 1.00 \\
\hline $\mathrm{CID}(\mathrm{A} 11 \mid \mathrm{A} 32)$ & $0.113(0.071)$ & 0.86 & $0.123(0.050)$ & 1.00 & $0.133(0.038)$ & 1.00 \\
\hline $\mathrm{CID}(\mathrm{A} 11 \mid \mathrm{B})$ & $0.028(0.037)$ & 0.06 & $0.016(0.017)$ & 0.13 & $0.012(0.008)$ & 0.16 \\
\hline $\mathrm{CID}(\mathrm{A} 21 \mid \mathrm{A} 11)$ & $0.194(0.061)$ & 1.00 & $0.202(0.051)$ & 1.00 & $0.231(0.030)$ & 1.00 \\
\hline $\mathrm{pCID}(\mathrm{A} 11 \mid \mathrm{A} 22 ; \mathrm{A} 21)$ & $0.078(0.043)$ & 0.74 & $0.082(0.050)$ & 0.96 & $0.084(0.030)$ & 1.00 \\
\hline $\mathrm{pCID}(\mathrm{A} 11 \mid \mathrm{A} 31 ; \mathrm{A} 21)$ & $0.036(0.032)$ & 0.22 & $0.030(0.023)$ & 0.55 & $0.017(0.017)$ & 0.83 \\
\hline $\mathrm{pCID}(\mathrm{A} 11 \mid \mathrm{A} 32 ; \mathrm{A} 21)$ & $0.031(0.032)$ & 0.19 & $0.022(0.021)$ & 0.40 & $0.012(0.016)$ & 0.72 \\
\hline $\mathrm{pCID}(\mathrm{A} 11 \mid \mathrm{B} ; \mathrm{A} 21)$ & $0.028(0.035)$ & 0.04 & $0.008(0.019)$ & 0.09 & $-0.010(0.015)$ & 0.09 \\
\hline $\mathrm{pCID}(\mathrm{A} 21 \mid \mathrm{A} 22 ; \mathrm{A} 11)$ & $0.036(0.031)$ & 0.19 & $0.021(0.022)$ & 0.33 & $0.009(0.014)$ & 0.61 \\
\hline $\mathrm{pCID}(\mathrm{A} 22 \mid \mathrm{A} 11 ; \mathrm{A} 21)$ & $0.065(0.045)$ & 0.26 & $0.068(0.036)$ & 0.69 & $0.063(0.025)$ & 0.99 \\
\hline $\mathrm{pCID}(\mathrm{A} 21 \mid \mathrm{A} 31 ; \mathrm{A} 11, \mathrm{~A} 22)$ & $0.119(0.045)$ & 0.96 & $0.124(0.040)$ & 1.00 & $0.128(0.028)$ & 1.00 \\
\hline $\mathrm{pCID}(\mathrm{A} 21 \mid \mathrm{A} 32 ; \mathrm{A} 11, \mathrm{~A} 22)$ & $0.090(0.047)$ & 0.78 & $0.093(0.044)$ & 0.99 & $0.095(0.024)$ & 1.00 \\
\hline $\mathrm{pCID}(\mathrm{A} 21 \mid \mathrm{B} ; \mathrm{A} 11, \mathrm{~A} 22)$ & $0.037(0.032)$ & 0.07 & $0.025(0.019)$ & 0.08 & $0.007(0.017)$ & 0.12 \\
\hline $\mathrm{pCID}(\mathrm{A} 11 \mid \mathrm{A} 31 ; \mathrm{A} 21, \mathrm{~A} 22)$ & $0.034(0.024)$ & 0.12 & $0.026(0.023)$ & 0.33 & $0.017(0.015)$ & 0.68 \\
\hline $\mathrm{pCID}(\mathrm{A} 22 \mid \mathrm{A} 31 ; \mathrm{A} 11, \mathrm{~A} 21)$ & $0.016(0.020)$ & 0.00 & $0.012(0.014)$ & 0.01 & $0.005(0.012)$ & 0.01 \\
\hline $\mathrm{pCID}(\mathrm{A} 31 \mid \mathrm{A} 21 ; \mathrm{A} 11, \mathrm{~A} 22)$ & $0.111(0.055)$ & 0.77 & $0.112(0.039)$ & 0.99 & $0.125(0.022)$ & 1.00 \\
\hline $\mathrm{pCID}(\mathrm{A} 21 \mid \mathrm{A} 32 ; \mathrm{A} 11, \mathrm{~A} 22, \mathrm{~A} 31)$ & $0.048(0.038)$ & 0.29 & $0.056(0.030)$ & 0.88 & $0.058(0.024)$ & 1.00 \\
\hline $\mathrm{pCID}(\mathrm{A} 21 \mid \mathrm{B} ; \mathrm{A} 11, \mathrm{~A} 22, \mathrm{~A} 31)$ & $0.033(0.029)$ & 0.03 & $0.022(0.015)$ & 0.08 & $0.009(0.019)$ & 0.17 \\
\hline $\mathrm{pCID}(\mathrm{A} 11 \mid \mathrm{A} 32 ; \mathrm{A} 21, \mathrm{~A} 22, \mathrm{~A} 31)$ & $0.021(0.021)$ & 0.01 & $0.016(0.016)$ & 0.03 & $0.010(0.010)$ & 0.14 \\
\hline $\mathrm{pCID}(\mathrm{A} 22 \mid \mathrm{A} 32 ; \mathrm{A} 11, \mathrm{~A} 21, \mathrm{~A} 31)$ & $0.024(0.021)$ & 0.01 & $0.015(0.020)$ & 0.02 & $0.013(0.013)$ & 0.02 \\
\hline pCID(A31|A32; A11, A21, A22) & $0.032(0.026)$ & 0.08 & $0.030(0.021)$ & 0.18 & $0.026(0.015)$ & 0.41 \\
\hline $\mathrm{pCID}(\mathrm{A} 32 \mid \mathrm{A} 21 ; \mathrm{A} 11, \mathrm{~A} 22, \mathrm{~A} 31)$ & $0.041(0.037)$ & 0.02 & $0.045(0.022)$ & 0.12 & $0.049(0.017)$ & 0.59 \\
\hline $\mathrm{pCID}(\mathrm{A} 11 \mid \mathrm{B} ; \mathrm{A} 21, \mathrm{~A} 22, \mathrm{~A} 31, \mathrm{~A} 32)$ & $0.038(0.036)$ & 0.02 & $0.028(0.022)$ & 0.15 & $0.018(0.018)$ & 0.12 \\
\hline $\mathrm{pCID}(\mathrm{A} 21 \mid \mathrm{B} ; \mathrm{A} 11, \mathrm{~A} 22, \mathrm{~A} 31, \mathrm{~A} 32)$ & $0.026(0.023)$ & 0.03 & $0.021(0.016)$ & 0.14 & $0.011(0.014)$ & 0.22 \\
\hline $\mathrm{pCID}(\mathrm{A} 22 \mid \mathrm{B} ; \mathrm{A} 11, \mathrm{~A} 21, \mathrm{~A} 31, \mathrm{~A} 32)$ & $0.058(0.030)$ & 0.03 & $0.047(0.023)$ & 0.09 & $0.040(0.018)$ & 0.12 \\
\hline $\mathrm{pCID}(\mathrm{A} 31 \mid \mathrm{B} ; \mathrm{A} 11, \mathrm{~A} 21, \mathrm{~A} 22, \mathrm{~A} 32)$ & $0.040(0.026)$ & 0.04 & $0.031(0.022)$ & 0.17 & $0.022(0.017)$ & 0.14 \\
\hline $\mathrm{pCID}(\mathrm{A} 32 \mid \mathrm{B} ; \mathrm{A} 11, \mathrm{~A} 21, \mathrm{~A} 22, \mathrm{~A} 31)$ & $0.045(0.032)$ & 0.04 & $0.039(0.022)$ & 0.14 & $0.029(0.020)$ & 0.18 \\
\hline $\mathrm{CID}(\mathrm{B} \mid \mathrm{A} 11)$ & $0.027(0.029)$ & 0.08 & $0.017(0.016)$ & 0.07 & $0.012(0.010)$ & 0.10 \\
\hline $\mathrm{CID}(\mathrm{B} \mid \mathrm{A} 21)$ & $0.022(0.023)$ & 0.06 & $0.014(0.013)$ & 0.04 & $0.010(0.007)$ & 0.08 \\
\hline $\mathrm{CID}(\mathrm{B} \mid \mathrm{A} 22)$ & $0.019(0.022)$ & 0.03 & $0.011(0.012)$ & 0.05 & $0.008(0.006)$ & 0.04 \\
\hline $\mathrm{CID}(\mathrm{B} \mid \mathrm{A} 31)$ & $0.020(0.024)$ & 0.08 & $0.013(0.015)$ & 0.08 & $0.008(0.009)$ & 0.11 \\
\hline $\mathrm{CID}(\mathrm{B} \mid \mathrm{A} 32)$ & $0.019(0.016)$ & 0.05 & $0.013(0.017)$ & 0.11 & $0.008(0.006)$ & 0.09 \\
\hline
\end{tabular}


associated node were much larger than $\mathrm{CID}(\mathrm{A} 11 \mid \mathrm{B})$ 's to the unassociated $\mathrm{B}$. Also, it could be observed that, on average, $\mathrm{CID}(\mathrm{A} 11 \mid \mathrm{A} 21)>\mathrm{CID}(\mathrm{A} 11 \mid \mathrm{A} 22)$, $\mathrm{CID}(\mathrm{A} 11 \mid \mathrm{A} 31)>\mathrm{CID}(\mathrm{A} 11 \mid \mathrm{A} 32)$, and $\mathrm{CID}(\mathrm{A} 11 \mid \mathrm{A} 21)$ $>\max (\mathrm{CID}(\mathrm{A} 11 \mid \mathrm{A} 31), \mathrm{CID}(\mathrm{A} 11 \mid \mathrm{A} 32))$. The order of the average CID values followed the order of association strengths of the nodes to A11 (Fig. 3a). Therefore, a CID value can not only distinguish the existence of an association but also reflect the strength of the association and successfully pick the direct (or strongest) association among all possible connections. On the other hand, since $100 \%$ of the pCID(A21|A31; A11) values were significant at $\alpha=0.05$ and the medians of pCID(A21|A31; A11) were the largest in different sample sizes, A31 was the most likely to be selected after A21 eliminating the effects from A11. For $\mathrm{N}=25$, A22 was more likely selected after A31 and A21 [63\% of the pCID(A11|A22; A21, A31) values were significant and pCID(A11|A22; A21, A31) had the largest median]. A32 [29\% of the pCID(A21|A32; A11, A22, A31) values were significant and $\mathrm{pCID}(\mathrm{A} 21 \mid \mathrm{A} 32 ; \mathrm{A} 11, \mathrm{~A} 22$, A31) had the largest median] might be selected as the last node associated with A11. With similar arguments, for $\mathrm{N}=50, \mathrm{~A} 21, \mathrm{~A} 31, \mathrm{~A} 32$, and A22 were consecutively identified; for $\mathrm{N}=100, \mathrm{~A} 21, \mathrm{~A} 31, \mathrm{~A} 22$, and A32 were consecutively identified.

However, the false networks were built spontaneously without consensus. All of the false networks that started from $B$ of the same combination of nodes only appeared less than or equal to five times in 100 simulations for $\mathrm{N}=25,50$, and 100. Therefore, the CID/pCID method robustly identified the relationships between nodes and the extended the association network. The asymmetric property of the CID and PCID was utilized to infer causal effects in the network. When $\operatorname{CID}(\mathrm{Y} \mid \mathrm{X})$ was more significant than $\mathrm{CID}(\mathrm{X} \mid \mathrm{Y})$ or when $\mathrm{pCID}(\mathrm{Y} \mid \mathrm{X} ; \mathrm{Z})$ was more significant than $\mathrm{pCID}(\mathrm{X} \mid \mathrm{Y} ; \mathrm{Z})$, Y was claimed to be the source of the relationship between $\mathrm{X}$ and $\mathrm{Y}$. In Fig. 3b-d, the numbers pointing in the correct directions are shown beside the arrows outside of the parentheses, whereas the numbers pointing in the incorrect directions are shown inside the parentheses. More than $90 \%$ of the significant A21-A32 and A11-A22 connections were with correct directions. Although the A11-A21 and A21-A31 associations were identified in more than $85 \%$ of the simulations for all the sample sizes, the percentages of arrows pointing in the correct directions might have been as few as $44 \%$ ( $\mathrm{A} 21-\mathrm{A} 31$ for $\mathrm{N}=100)$. The simulation results implied that a large sample size would aggravate the confusion regarding causality. For example, while 71 out of $86(82.6 \%)$ arrows from $\mathrm{A} 21$ pointed to $\mathrm{A} 31$ for $\mathrm{N}=25$ and 67 out of $99(67.7 \%)$ arrows from A21 pointed to A31 for $\mathrm{N}=50$, only 44 out of 100 (44.0\%) arrows from
A21 pointed to A31 for $\mathrm{N}=100$. We conjecture that the strong association between A11 and A21 would disguise the cause-effect relationship between them.

\section{Literature confirmed the results of CBF-COR pathway reconstruction in Arabidopsis}

$\mathrm{C}$-repeat binding factors $(\mathrm{CBF})$ bind to the promoter regions of downstream cold-regulated (COR) genes and induce COR genes expression under cold stress (Fowler and Thomashow 2002; McKhann et al. 2008; Doherty et al. 2009; Zhao et al. 2016). A heatmap and cluster analysis of the expression fold changes of CBF and COR genes at different time points after cold treatment relative to their corresponding control samples is shown in Fig. 6. The expressions of the CBF genes under cold stress increased earlier than those of the COR genes in both root and shoot tissues. Among them, CBF3 had the highest relative expressions from 0.5 to $12 \mathrm{~h}(\mathrm{~s})$ in root tissues and from 1 to $12 \mathrm{~h}(\mathrm{~s})$ in shoot tissues; this was reflected in the outcome that $C B F 3$ was identified as the primary inducer of COR genes in our CID/pCID network results (Fig. 4a). In fact, it was evidenced that COR47, COR78, COR15A, COR15B, and COR6.6 can be activated by $C B F 3$ under cold stress (Sakuma et al. 2006). The target genes, COR47 and COR6.6, had similar expression levels, while COR15A and COR15B had similar expression levels. The CBF-COR GRN reconstructed by $\mathrm{CID} / \mathrm{pCID}$ reflected their similarities by linking COR47-COR6.6 and COR15A-COR15B. In particular, in root samples, the expressions of COR78 were induced as early as $6 \mathrm{H}$ after cold treatment; it reacted before the other COR genes.

Experiments based on transgenic plants constitutively expressing $C B F 1, C B F 2$, and $C B F 3$ have suggested that overexpression of the three genes induces the expression of similar gene sets, including COR47, COR6.6, and COR78 (Gilmour et al. 2004). RNA blot analysis has been conducted by others to confirm that the overexpression of $C B F 1$ and $C B F 3$ would induce COR15A, COR78, COR47, and COR6.6 gene expressions (Kasuga et al. 1999; Taji et al. 2002; Seki et al. 2002; Fowler and Thomashow 2002). McKhann et al. (2008) reported that the expression of COR15B may last for 5 weeks after cold treatment, while COR47 was only expressed within $24 \mathrm{~h}$ after cold treatment. The expression patterns of the microarray data investigated in this study were consistent with their findings (Fig. 6); COR47 was set upstream relative to COR15B in the resulting network (Fig. 4a).

\section{Literature supported the discovered bHLH GRN in rice}

A family of bHLH (basic helix-loop-helix) transcription factors in plants plays a principal role in various developmental processes (Ding et al. 2009; Chen and 

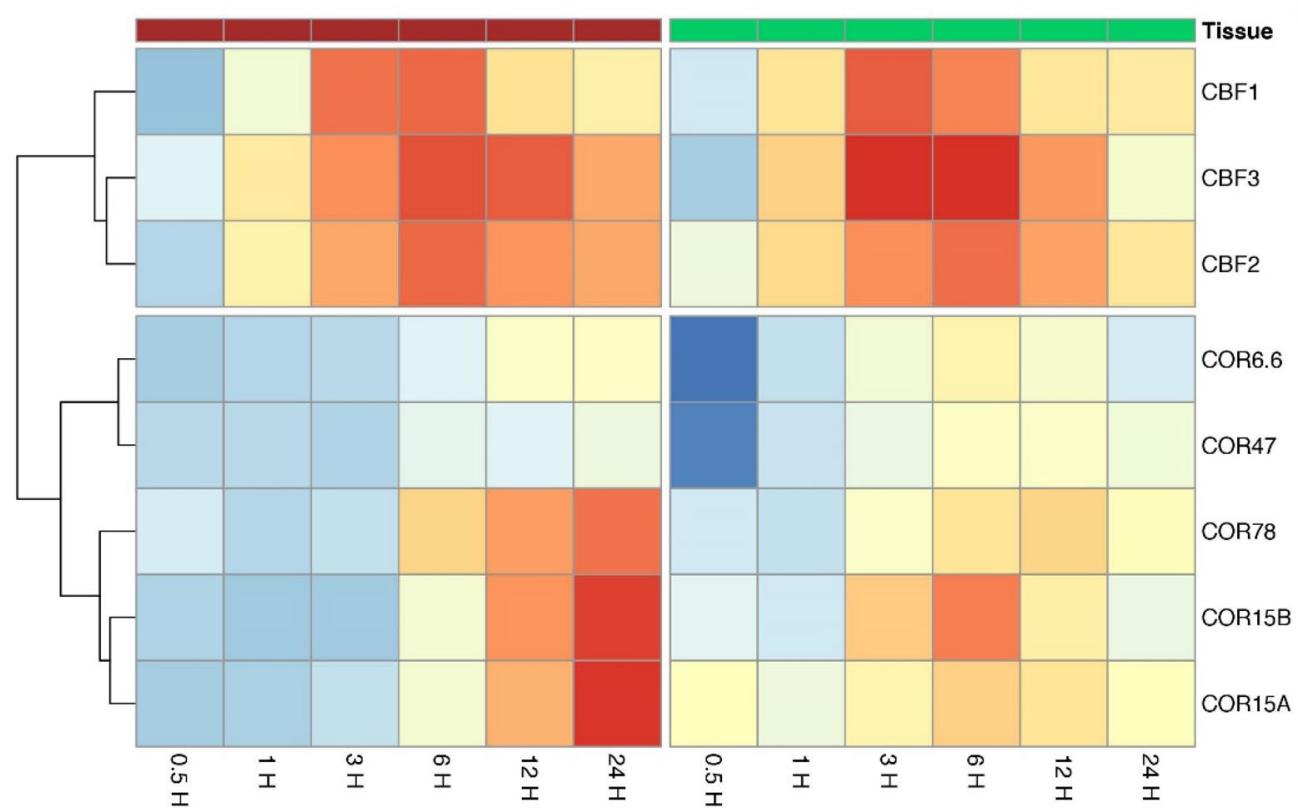

Fig. 6 A heatmap and cluster analysis of the expression fold changes of CBF and COR genes at different time points after cold treatment relative to their corresponding control samples

Chory 2011; Cui et al. 2016) that might be affected when plants suffer abiotic stresses. In this study, we explored the responses of the OsbHLH genes and their potential target genes under abiotic stresses. We combined all the resulting paths starting from all the sources to form the bHLH GRN (Fig. 5). The numbers in the node are the shortened ID numbers of the bHLH genes (for example, "001" stands for OsbHLH001 in rice). The resulting network involved 83 nodes and 107 edges, while some probes not connected to any other probe (nodes having "NA" for in-degree and outdegree in Additional file 2: Table S1) were excluded. The network obeys the power law of a biological network with an average degree of 2.58 (Additional file 1: Figure S2). The source having the largest out-degree value was OsbHLH104-2 (out-degree $=8$ ), and the target having the largest in-degree value was OsbHLH149-1 (in-degree=11). OsbHLH104-2 was also the most connected gene (in-degree + out-degree $=17$ ), while OsbHLH060 was the second most connected one (in-degree + out-degree $=15$ ).

According to a previous study, OsbHLH104 (LOC_ Os07g05010), a putative phytochrome-interacting factor (OsPIF14), binds to N-boxes [CACG(A/C)G] in the OsDREB1B promoter and represses OsDREB1B gene expression, which reduces freezing tolerance in rice (Cordeiro et al. 2016). Interestingly, according to another study, PIF3/AtbHLH008 (At1g09530), an OsPIF14/OsbHLH104 homolog repressing photomorphogenesis, also has a negative impact on freezing tolerance by directly down-regulating the expression of CBFs in Arabidopsis (Jiang et al. 2017). These previous studies have indicated that OsbHLH104 plays a pivotal role in the connection between light and stress signaling.

According to one study, OsbHLH060 (LOC_ Os08g04390), also known as OsPRI1 [POSITIVE REGULATOR OF IRON HOMEOSTASIS 1], directly activates the expression of OsIRO2 [iron-related transcription factor 2/OsbHLH056 (LOC_Os01g72370)] and OsIRO3 [OsbHLH063 (LOC_Os03g26210)], which mediates rice responses to Fe deficiency (Zhang et al. 2017). Similarly, another study has indicated that the AtbHLH034 (At3g23210), 104 (At4g14410), 105/ILR3 [IAACINE RESISTANT 3 (At5g54680)], and 115 (At1g51070) genes, which are highly homologous to the OsPRI1/ OsbHLH060 gene, directly activate the transcription of the Ib subgroup $b H L H$ genes, AtbHLH38/39/100/101 (At3g56970/At3g56980/At2g41240/At5g04150), and PYE [POPE $\underline{Y E / A t b H L H 047 ~(A t 3 g 47640), ~ a n ~ O s I R O 3 / ~}$ OsbHLH063 homolog], that regulate Fe homeostasis in Arabidopsis (Wang et al. 2007; Long et al. 2010; Zhang et al. 2015; Li et al. 2016; Liang et al. 2017). Moreover, AtbHLH104 has been found to positively regulate several heavy metal detoxification-associated genes, such as IREG2 (iron regulated 2), MTP3 (․etal tolerance protein 3), HMA3 (heavy metal ATPase 3), and NAS4 (nicotianamine synthase 4), which confer tolerance to cadmium stress in Arabidopsis (Yao et al. 2018). These 
previous studies suggest the possibility that OsbHLH060 is involved in cross-talk between Fe homeostasis and Cd stress tolerance.

\section{Utilization of CID/pCID on modern transcriptomic data}

In this study, we demonstrated the construction of the GRN using microarray data. The main reason using microarray data is that after more than two decades of accumulation in the database, there are enough microarray expression samples for network construction. According to the simulation results, when the sample size is as small as 25 or 50 , more than $50 \%$ of the resulting networks recovered only upstream regulatory events in the real network. Along with the advance of biotechnology, measuring global gene expression profiles by the whole transcriptome shotgun sequencing (RNA-seq) and single cell RNA-seq (scRNAseq) are common practice nowadays. It can be expect to have adequate sequencing data in the near future for network construction. It is worthwhile to mention that the gene expressions by sequencing are present by non-negative integers called 'read counts'. The read counts are not normally distributed. Instead, the read counts are commonly analyzed as random samples from a Poisson or a negative binomial distribution (Robinson et al. 2010; Love et al. 2014, p. 2). The CID/pCID independent of distributional assumptions can be directly applied to sequencing data without a doubt. The non-distribution assumption of CID/pCID also implies the possibilities of applying CID/pCID on integrated transcriptomic, proteomic, metabolic, phenotypic data of different formats to construct bipartite or multipartite networks (Bass et al. 2013).

\section{Conclusion}

Rapidly accumulated publicly accessible gene expression datasets have made it possible to systematically construct gene regulatory networks. In this study, we adopted a diverse dataset collected under different abiotic stresses. This strategy not only increased the sample size for statistical analysis but also made it possible to capture the gene-gene interactions under various circumstances simultaneously. Surely different combinations of gene expression datasets can be selected to better represent the population of interest based on the research purposes.

The proposed method makes use of the asymmetry of CID/pCID to determine the path directions in the gene regulatory network. The directions inferred in this study were then partly verified through literature reviews, although more finely designed experiments must be performed to piece together more solid biological evidence. In this study, we nonetheless demonstrated an exhaustive search in the simulation as well as heuristic methods in real datasets to accelerate the computation. The heuristic approach applied to the bHLH genes adopted as many resource/target bHLH genes as possible to demonstrate a mechanical way to build a comprehensive network. One can also pick fewer transcription factors or genes of interest in order to conduct an exhaustive search on a smaller scale.

In conclusion, this study proposed a three-step procedure to construct a directed gene regulatory network starting from the identification of incorporated genes connected as local pathways. The method is potentially applicable for deciphering causal events in proteomics, metabolomics, and epigenomics. Biologists can also customize the desired complexity of the inferred networks based on the complexity of the investigated biological systems. This flexible and constructive method may help to efficiently decipher gene regulatory pathways and achieve higher predictive power in practical applications.

\section{Supplementary information}

Supplementary information accompanies this paper at https://doi. org/10.1186/s40529-019-0268-8.

\begin{abstract}
Additional file 1: Figure S1. Summary of the networks reconstructed in the simulations for $\mathrm{N}=$ (A) 25, (B) 50, and (C) 100. Networks consisting of the same set of nodes were grouped together. Only groups occurred at least 5 times are shown. Figure S2. The scatter plot of the log(total degree) and the log(frequency) in the bHLH gene regulatory network. The inversely proportional trend between the log(total degree) and the $\log$ (frequency) indicates the resulting network obeys the power law.

Additional file 2: Table S1. The list of $122 \mathrm{bHLH}$-related probess in the microarray. The ones recognize the G-box in their targets' promoter regions (Gonzalez 2015) are classified as the 'Source' genes; the ones containing G-box sequences in their promoter regions are classified as the 'Target' genes. 'in.degree' is the number of directed edge(s) using the probe as the target; 'out.degree' is the number of directed edge(s) using the probe as the source; 'total.degree' $=$ 'in.degree' + 'out.degree'. Table S2. The resulting sub-networks for bHLH source probes.
\end{abstract}

\section{Abbreviations}

bHLH: basic helix-loop-helix; CBF: C-repeat binding factor; CID: the coefficient of intrinsic dependence; COR: cold-regulated gene; GSAA: gene set association analysis; GRN: gene regulatory network; NCBI-GEO: National Center for Biotechnology Information-Gene Expression Omnibus; PCC: Pearson correlation coefficient; pCID: partial coefficient of intrinsic dependence; pPCC: partial Pearson correlation coefficient; RAP-DB: Rice Annotation Project Database; RMA: robust multichip average; TAIR: the Arabidopsis Information Resource; TF: transcription factor.

\section{Authors' contributions}

LYDL drafted the manuscript, initiated the research, revised the manuscript, and provided funding for the study. YCH performed most of the analysis. HCC, YWY, and MCC validated the inferred gene regulatory events. All authors read and approved the final manuscript.

\section{Funding}

This research is supported by Ministry of Science and Technology in Taiwan, ROC (Grant No. MOST 108-2313-B-002-050). 


\section{Availability of data and materials}

The microarray experiments in this study can be downloaded from the Arabidopsis Information Resource (TAIR) database (https://www.arabidopsi s.org/servlets/Search?type=expr\&search_action=new_search) (submission number: ME00325), and the NCBI-GEO database (https://www.ncbi.nlm.nih. gov/geo/) (accession numbers: GSE6901 and GSE14275).

\section{Ethics approval and consent to participate}

Not applicable.

\section{Consent for publication}

Not applicable.

\section{Competing interests}

The authors declare that they have no competing interests.

\section{Author details}

${ }^{1}$ Department of Agronomy, National Taiwan University, Taipei 106, Taiwan.

${ }^{2}$ Department of Horticulture and Landscape Architecture, National Taiwan University, Taipei 106, Taiwan.

Received: 9 May 2019 Accepted: 17 August 2019

Published online: 11 September 2019

\section{References}

Baldoni E, Genga A, Cominelli E (2015) Plant MYB transcription factors: their role in drought response mechanisms. Int J Mol Sci 16:15811-15851. https://doi.org/10.3390/ijms160715811

Bass JIF, Diallo A, Nelson J et al (2013) Using networks to measure similarity between genes: association index selection. Nat Methods 10:1169-1176. https://doi.org/10.1038/nmeth.2728

Chen M, Chory J (2011) Phytochrome signaling mechanisms and the control of plant development. Trends Cell Biol 21:664-671. https://doi. org/10.1016/j.tcb.2011.07.002

Cordeiro AM, Figueiredo DD, Tepperman J et al (2016) Rice phytochromeinteracting factor protein OsPIF14 represses OsDREB1B gene expression through an extended $\mathrm{N}$-box and interacts preferentially with the active form of phytochrome B. Biochim Biophys Acta BBA Gene Regul Mech 1859:393-404. https://doi.org/10.1016/j.bbagrm.2015.12.008

Cui J, You C, Zhu E et al (2016) Feedback regulation of DYT1 by interactions with downstream bHLH factors promotes DYT1 nuclear localization and anther development. Plant Cell 28:1078-1093. https://doi.org/10.1105/ tpc.15.00986

de la Fuente A, Bing N, Hoeschele I, Mendes P (2004) Discovery of meaningful associations in genomic data using partial correlation coefficients. Bioinformatics 20:3565-3574. https://doi.org/10.1093/bioinformatics/bth445

Ding W, Yu Z, Tong Y et al (2009) A transcription factor with a bHLH domain regulates root hair development in rice. Cell Res 19:1309-1311. https:// doi.org/10.1038/cr.2009.109

Doherty CJ, Van Buskirk HA, Myers SJ, Thomashow MF (2009) Roles for Arabidopsis CAMTA transcription factors in cold-regulated gene expression and freezing tolerance. Plant Cell 21:972-984. https://doi.org/10.1105/ tpc.108.063958

Edgar R, Domrachev M, Lash AE (2002) Gene Expression Omnibus: NCBI gene expression and hybridization array data repository. Nucleic Acids Res 30:207-210

Fowler S, Thomashow MF (2002) Arabidopsis transcriptome profiling indicates that multiple regulatory pathways are activated during cold acclimation in addition to the CBF cold response pathway. Plant Cell 14:1675-1690. https://doi.org/10.1105/tpc.003483

Fujita M, Fujita Y, Noutoshi Y et al (2006) Crosstalk between abiotic and biotic stress responses: a current view from the points of convergence in the stress signaling networks. Curr Opin Plant Biol 9:436-442. https://doi. org/10.1016/j.pbi.2006.05.014

Garcia-Hernandez M, Berardini T, Chen G et al (2002) TAIR: a resource for integrated Arabidopsis data. Funct Integr Genomics 2:239-253. https://doi. org/10.1007/s10142-002-0077-z

Gilmour SJ, Fowler SG, Thomashow MF (2004) Arabidopsis transcriptional activators CBF1, CBF2, and CBF3 have matching functional activities.
Plant Mol Biol 54:767-781. https://doi.org/10.1023/B:PLAN.0000040902 $.06881 . \mathrm{d} 4$

Gomez-Casati DF, Zanor MI, Busi MV (2013) Metabolomics in plants and humans: applications in the prevention and diagnosis of diseases. Biomed Res Int. https://doi.org/10.1155/2013/792527

Gonzalez DH (2015) Plant transcription factors: evolutionary, structural and functional aspects. Academic Press, Cambridge

Hayfield T, Racine JS (2008) Nonparametric econometrics: the np package. J Stat Softw 27:5

Higa CHA, Hashimoto RF, Hirata R, et al (2009) Inference of gene regulatory network using temporal coefficient of determination obtained from ergodic Markov chains. In: 2009 IEEE International Workshop on Genomic Signal Processing and Statistics. pp 1-4

Hsiao Y-C, Liu L-YD (2016) A stepwise approach of finding dependent variables via coefficient of intrinsic dependence. J Comput Biol 23:42-55

Hsing T, Liu L-Y, Brun M, Dougherty ER (2005) The coefficient of intrinsic dependence (feature selection using el CID). Pattern Recognit 38:623-636. https://doi.org/10.1016/j.patcog.2004.09.002

Irizarry RA, Hobbs B, Collin F et al (2003) Exploration, normalization, and summaries of high density oligonucleotide array probe level data. Biostatistics 4:249-264. https://doi.org/10.1093/biostatistics/4.2.249

Jiang B, Shi Y, Zhang X et al (2017) PIF3 is a negative regulator of the CBF pathway and freezing tolerance in Arabidopsis. Proc Natl Acad Sci 114:E6695-E6702. https://doi.org/10.1073/pnas.1706226114

Karlebach G, Shamir R (2008) Modelling and analysis of gene regulatory networks. Nat Rev Mol Cell Biol 9:770-780. https://doi.org/10.1038/ nrm2503

Kasuga M, Liu Q, Miura S et al (1999) Improving plant drought, salt, and freezing tolerance by gene transfer of a single stress-inducible transcription factor. Nat Biotechnol 17:287-291. https://doi. org/10.1038/7036

Kiribuchi K, Jikumaru Y, Kaku H et al (2005) Involvement of the basic helixloop-helix transcription factor RERJ1 in wounding and drought stress responses in rice plants. Biosci Biotechnol Biochem 69:1042-1044. https ://doi.org/10.1271/bbb.69.1042

Knight H, Knight MR (2001) Abiotic stress signalling pathways: specificity and cross-talk. Trends Plant Sci 6:262-267. https://doi.org/10.1016/S1360 -1385(01)01946-X

Le Novère N (2015) Quantitative and logic modelling of molecular and gene networks. Nat Rev Genet 16:146-158. https://doi.org/10.1038/nrg3885

Li X, Duan X, Jiang H et al (2006) Genome-wide analysis of basic/helix-loophelix transcription factor family in rice and Arabidopsis. Plant Physiol 141:1167-1184. https://doi.org/10.1104/pp.106.080580

Li X, Zhang H, Ai Q et al (2016) Two bHLH transcription factors, bHLH34 and bHLH104, regulate iron homeostasis in Arabidopsis thaliana. Plant Physiol 170:2478-2493. https://doi.org/10.1104/pp.15.01827

Liang G, Zhang H, Li X et al (2017) bHLH transcription factor bHLH1 15 regulates iron homeostasis in Arabidopsis thaliana. J Exp Bot 68:1743-1755. https://doi.org/10.1093/jxb/erx043

Liseron-Monfils C, Ware D (2015) Revealing gene regulation and associations through biological networks. Curr Plant Biol 3-4:30-39. https://doi. org/10.1016/j.cpb.2015.11.001

Liu LD (2005) Coefficient of intrinsic dependence: a new measure of association. Texas A\&M University, College Station

Liu L-YD, Chen C-Y, Chen M-JM et al (2009) Statistical identification of gene association by CID in application of constructing ER regulatory network. BMC Bioinform 10:85. https://doi.org/10.1186/1471-2105-10-85

Liu L-YD, Chang L-Y, Kuo W-H et al (2012) In silico prediction for regulation of transcription factors on their shared target genes indicates relevant clinical implications in a breast cancer population. Cancer Inform 11:113-137. https://doi.org/10.4137/CIN.S8470

Long TA, Tsukagoshi H, Busch W et al (2010) The bHLH transcription factor POPEYE regulates response to iron deficiency in Arabidopsis roots. Plant Cell 22:2219-2236. https://doi.org/10.1105/tpc.110.074096

Love MI, Huber W, Anders S (2014) Moderated estimation of fold change and dispersion for RNA-seq data with DESeq2. Genome Biol 15:550. https:// doi.org/10.1186/s13059-014-0550-8

Mantione KJ, Kream RM, Kuzelova H et al (2014) Comparing bioinformatic gene expression profiling methods: microarray and RNA-seq. Med Sci Monit Basic Res 20:138-141. https://doi.org/10.12659/MSMBR.892101 
McKhann HI, Gery C, Bérard A et al (2008) Natural variation in CBF gene sequence, gene expression and freezing tolerance in the Versailles core collection of Arabidopsis thaliana. BMC Plant Biol 8:105. https://doi. org/10.1186/1471-2229-8-105

Nakamura J, Yuasa T, Huong TT et al (2011) Rice homologs of inducer of CBF expression (OsICE) are involved in cold acclimation. Plant Biotechnol 28:303-309. https://doi.org/10.5511/plantbiotechnology.11.0421a

Pérez-de-Castro AM, Vilanova S, Cañizares J et al (2012) Application of genomic tools in plant breeding. Curr Genomics 13:179-195. https://doi. org/10.2174/138920212800543084

Pilpel Y, Sudarsanam P, Church GM (2001) Identifying regulatory networks by combinatorial analysis of promoter elements. Nat Genet 29:153-159. https://doi.org/10.1038/ng724

Ritchie ME, Phipson B, Wu D et al (2015) limma powers differential expression analyses for RNA-sequencing and microarray studies. Nucleic Acids Res. https://doi.org/10.1093/nar/gkv007

Robinson MD, McCarthy DJ, Smyth GK (2010) edgeR: a bioconductor package for differential expression analysis of digital gene expression data. Bioinformatics 26:139-140. https://doi.org/10.1093/bioinformatics/btp616

Rykunov D, Beckmann ND, Li H et al (2016) A new molecular signature method for prediction of driver cancer pathways from transcriptional data. Nucleic Acids Res 44:e110. https://doi.org/10.1093/nar/gkw269

Sakai H, Lee SS, Tanaka T et al (2013) Rice Annotation Project Database (RAPDB): an integrative and interactive database for rice genomics. Plant Cell Physiol 54:e6. https://doi.org/10.1093/pcp/pcs183

Sakuma Y, Maruyama K, Osakabe Y et al (2006) Functional analysis of an Arabidopsis transcription factor, DREB2A, involved in drought-responsive gene expression. Plant Cell 18:1292-1309. https://doi.org/10.1105/ tpc.105.035881

Schaller GE (2012) Ethylene and the regulation of plant development. BMC Biol 10:9. https://doi.org/10.1186/1741-7007-10-9

Segal E, Friedman N, Kaminski N et al (2005) From signatures to models: understanding cancer using microarrays. Nat Genet 37:S38-S45. https:// doi.org/10.1038/ng1561

Seki M, Narusaka M, Kamiya A et al (2002) Functional annotation of a fulllength Arabidopsis cDNA collection. Science 296:141-145. https://doi org/10.1126/science.1071006

Seo J-S, Joo J, Kim M-J et al (2011) OsbHLH148, a basic helix-loop-helix protein, interacts with OsJAZ proteins in a jasmonate signaling pathway leading to drought tolerance in rice. Plant J 65:907-921. https://doi.org/10.1111/ j.1365-313X.2010.04477.x

Simcha DM, Younes L, Aryee MJ, Geman D (2013) Identification of direction in gene networks from expression and methylation. BMC Syst Biol 7:118. https://doi.org/10.1186/1752-0509-7-118
Song L, Langfelder P, Horvath S (2012) Comparison of co-expression measures: mutual information, correlation, and model based indices. BMC Bioinform 13:328. https://doi.org/10.1186/1471-2105-13-328

Taji T, Ohsumi C, luchi S et al (2002) Important roles of drought- and coldinducible genes for galactinol synthase in stress tolerance in Arabidopsis thaliana. Plant J Cell Mol Biol 29:417-426

Todaka D, Nakashima K, Shinozaki K, Yamaguchi-Shinozaki K (2012) Toward understanding transcriptional regulatory networks in abiotic stress responses and tolerance in rice. Rice 5:6. https://doi. org/10.1186/1939-8433-5-6

Tsai C-A, Liu L-YD (2013) Identifying gene set association enrichment using the coefficient of intrinsic dependence. PLoS ONE 8:e58851. https://doi. org/10.1371/journal.pone.0058851

Wang H-Y, Klatte M, Jakoby M et al (2007) Iron deficiency-mediated stress regulation of four subgroup lb BHLH genes in Arabidopsis thaliana. Planta 226:897-908. https://doi.org/10.1007/s00425-007-0535-x

Yao X, Cai Y, Yu D, Liang G (2018) bHLH104 confers tolerance to cadmium stress in Arabidopsis thaliana. J Integr Plant Biol 60:691-702. https://doi. org/10.1111/jipb.12658

Zhang J, Liu B, Li M et al (2015) The bHLH transcription factor bHLH104 interacts with IAA-LEUCINE RESISTANT3 and modulates iron homeostasis in Arabidopsis. Plant Cell 27:787-805. https://doi.org/10.1105/tpc.114.13270

Zhang H, Li Y, Yao X et al (2017) Positive regulator of iron homeostasis1, OsPRI1, facilitates iron homeostasis. Plant Physiol 175:543-554. https://doi. org/10.1104/pp.17.00794

Zhao C, Zhang Z, Xie S et al (2016) Mutational evidence for the critical role of CBF transcription factors in cold acclimation in Arabidopsis. Plant Physiol 171:2744-2759. https://doi.org/10.1104/pp.16.00533

\section{Publisher's Note}

Springer Nature remains neutral with regard to jurisdictional claims in published maps and institutional affiliations.

\section{Submit your manuscript to a SpringerOpen ${ }^{\circ}$ journal and benefit from:}

- Convenient online submission

- Rigorous peer review

- Open access: articles freely available online

- High visibility within the field

Retaining the copyright to your article

Submit your next manuscript at springeropen.com 